\title{
Harnessing beliefs to optimally disclose contestants' types
}

\section{Marco Serena ${ }^{1}$}

Received: 2 October 2017 / Accepted: 9 July 2021 / Published online: 23 July 2021

(C) The Author(s) 2021, corrected publication 2021

\begin{abstract}
A contestant's effort depends on her knowledge of her rival's type. This knowledge is often limited in real-life contests. We propose a model where the principal of a contest has commitment power to verifiably disclose contestants' types. We investigate the optimal disclosure policy to stimulate contestants' efforts. Full disclosure stimulates more (less) effort than full concealment if high-types are more (less) likely than lowtypes. However, regardless of the likelihood of types, the optimal policy is that of contingent disclosure; it is optimal to commit to disclosing if both contestants are high types and concealing otherwise.
\end{abstract}

Keywords Contests $\cdot$ Strategic complements $\cdot$ Strategic substitutes $\cdot$ Information JEL Classification C72 - D82

\begin{abstract}
This article is based on the first chapter of my Ph.D. dissertation at Universidad Carlos III, Madrid. Previous versions of this paper circulated under the name "Information in contests" in 2013-2015, and "Harnessing beliefs to stimulate efforts" in 2016-2018. The supervision of Luis Corchón is greatly appreciated. I am pleased to acknowledge useful comments by a Co-Editor, three reviewers, and Carmen Beviá, Guillermo Caruana, Robert Edwards, Tore Ellingsen, Christian Ewerhart, Natalia Fabra, Dawei Fang, Qiang Fu, Aart Gerritsen, Andrea Guariso, Angel Hernando-Veciana, René Kirkegaard, Harald Lang, Jingfeng Lu, Thomas Mariotti, Ricardo Martinez, Diego Moreno, Ron Siegel, Giancarlo Spagnolo, and Huseyin Yildirim. I would like to thank participants at the 2013 GSE Summer Forum (Pompeu Fabra), the 2013 Young Researchers MOVE Workshop on Contests and Tournaments (UAB), the First Microeconomics Graduate Workshop (CEMFI), the 2014 Economics PhD Conference (Warwick), the 25th International Conference on Game Theory (Stony Brook), the 2014 MACCI Enter Workshop (Mannheim), the 2014 European Winter Meeting of the Econometric Society (Carlos III), the 2015 ECARES Summer School (ULB), the 2015 SAET Conference (Cambridge), and the 2016 CBESS Conference on Contests (UEA). I acknowledge financial support from ECO2011-25330/ECON. All errors are my own.
\end{abstract}

Marco Serena

marco.serena@tax.mpg.de

1 Max Planck Institute for Tax Law and Public Finance, Munich, Germany 


\section{Introduction}

Running relative performance contests is a popular way to stimulate workers' efforts. It is estimated that roughly between one third (Loew 2015) and two thirds (The Wall Street Journal 2012) of firms use some such form of internal ranking. A manager can provide some information to the competing workers about each others' types, and competing workers do not a priori have this information on their rivals unless informed by the manager. Workers' types can be, for instance, their past performance, their inherent ability or even their identity itself. If the manager discloses types before the proclamation of the winner of the contest (i.e., the worker who obtains the promotion, the tenure, or the bonus), then such a disclosure affects workers' efforts, and consequently the firm's profit.

Analogous information disclosure problems arise in other settings. For instance, the organizer of a sports tournament might disclose information about players that would otherwise be too costly or impossible to retrieve by the players themselves; in chess tournaments, players' Elo ranking is sometimes displayed on the pairing-list board, and in the NBA fine-tuned data collected by advanced tracking cameras are disclosed online. Similarly, scholars compete for grants by submitting research projects, and they presumably do not know who they are competing against unless the grant-awarding entity publicly discloses the list of participants. The awareness of a rival's type affects a contestant's effort. For example, an average researcher aware of being shortlisted to compete for a grant against a leading scholar might give up hope and exert little effort. In the present paper we explore the effort-maximizing disclosure policy.

Our model has an informed principal (manager, sports tournament organizer, grantawarding entity) who maximizes the aggregate effort of two contestants (workers, players, scholars) who compete in a Tullock contest with discriminatory parameter $r \in(0,1]$ (Tullock 1980). First, the principal commits to a costless and verifiable disclosure policy about contestants' types (modeled as marginal costs of efforts). Then, nature assigns a type to every contestant. Contestants know their own type, but their knowledge of their rival's type depends on the principal's disclosure policy. ${ }^{1}$ Our model assumes that there are two contestants. This assumption admittedly stylizes the applications we described, but it allows us to abstract from contestants' endogenous participation decisions, and thus to isolate the effect of the disclosure policy on contestants' efforts. Each contestant is of high-type with probability $p \in[0,1]$, and low-type with the complementary probability. Types are drawn independently and $p$ is common knowledge. We find that the probability of high-types $p$ drives the optimal disclosure policy. We provide an intuitive explanation in what follows.

We describe two building blocks of our results. First, around the equilibrium of our setup the effort of the high- (low-) type and that of her rival are strategic complements

\footnotetext{
1 The principal commits to a disclosure policy ex-ante, that is, before observing types. This captures the idea that the principal chooses and commits to the rules of the contest, among which the disclosure policy, which are implemented every time the contest is run. This argument is similar to the one in Rayo and Segal (2010), where the precommitment power of the sender is a way to build long-run reputation in the eyes of the sequence of "short-run" receivers. In such a repeated game, losses of reputation would jeopardize the sender's long-run profits. Moreoever, in "Appendix B.1" we show that the principal is better off committing to her disclosure policy ex-ante, rather than ex-post, if she could choose.
} 
(substitutes). Second, the effort of a contestant, regardless of her type, is maximum when she is up against a contestant of the same type. We call this situation an even contest, and its effort the "stimulated effort," in contrast to an uneven contest and the "discouraged effort" where types differ. ${ }^{2}$

With these two building blocks in mind, consider the effect of the probability of high-types $p$ on the optimal disclosure policy. Trivially, in the extreme case of $p \in$ $\{0,1\}$, any attempt to conceal types in order to affect efforts is fruitless since contestants know $p$, and thus disclosure policies are all outcome-equivalent. We analyze the case of a high (low), but non-extreme, value of $p$ in Case I (II) below.

\section{Case I (high $p$ )}

Consider a high-type. Under disclosure, a high-type will exert the stimulated effort with high probability $(p)$ and the discouraged effort with low probability $(1-p)$. Under concealment, a high-type believes she is in an even (uneven) contest with high (low) probability, and knows that her most likely rival (a high-type) has the same beliefs. Thus, the resulting level of effort of the high-type under concealment is close to the effort that the principal expects from the high-type under disclosure, which in fact is the high-type's stimulated effort with high probability $(p)$ and the high-type's discouraged effort with low probability $(1-p)$.

Consider a low-type. Under disclosure, a low-type will exert the stimulated effort with low probability and the discouraged effort with high probability. Under concealment, a low-type believes she is most likely up against a high-type who believes she is most likely up against another high-type, and so on. Thus, a low-type under concealment, despite knowing she is most likely up against a high-type, exerts significantly less effort than in a high-vs-low contest under disclosure, because she knows that her (most likely) high-type rival believes that the contest is (most likely) even, rather than uneven. This discouragement of a low-type is obviously only present under concealment. For brevity, we call this discouragement caused by concealment when $p$ is high the negative $p$-Effect (hereafter, “- $p \mathrm{E}$ "), which is stronger the more $p$ is far from $1 / 2$ and close to 1 .

\section{Case II (low $p)$}

In a nutshell, everything said for Case I carries over by just swapping high- and lowtype, and changing "less" and "discouragement" into "more" and "encouragement." In fact, the key difference is that now the $p$-Effect is positive. For brevity, we call this encouragement caused by concealment when $p$ is low the positive $p$-Effect (hereafter, " $+p \mathrm{E}$ "), which is stronger the more $p$ is far from $1 / 2$ and close to 0 .

The present paper makes the case that the $p$-Effects drive the optimal disclosure policy in contests. In fact, we find that in a standard Tullock contest aggregate effort is maximized by disclosure when $p \in(1 / 2,1)$ in order to avoid the $-p \mathrm{E}$, and by concealment when $p \in(0,1 / 2)$ in order to make the most out of the $+p \mathrm{E}^{3}{ }^{3}$ Concealment and disclosure yield the same aggregate effort in the remaining cases; namely,

\footnotetext{
2 When two contestants are of the same type, they maximize the same payoff function, and this typically yields the maximum individual effort in standard two-player Tullock contests (see, e.g., Baik 1994; Nti 1999).

3 Recall that $+p \mathrm{E}$ and $-p \mathrm{E}$ are present only under concealment.
} 
the symmetric prior $p=1 / 2$, because $+p \mathrm{E}$ and $-p \mathrm{E}$ cancel out, and the degenerate prior $p \in\{0,1\}$. These are the results of Sect. 4 .

In Sect. 5, we consider a principal who can precommit to disclosing or concealing contestants' types contingently on the realization of types. We show that the optimal information disclosure, regardless of $p$, is to commit to disclosing contestants' types if contestants are both high-types and concealing all other realizations of types. Such an optimal contingent disclosure policy thus improves upon the full disclosure and full concealment policies considered in Sect. 4. The intuition behind this result relies again on $+p \mathrm{E}$ and $-p \mathrm{E}$, and it will be given in Sect. 5, after presenting the result.

After a discussion of the related literature in Sect. 2, the main body of the paper (Sects. 3-6) includes what is needed to understand the intuition behind the optimal disclosure policies, and everything else is moved to the appendices. In particular, Sect. 3 spells out the contest model and the disclosure game. Sections 4 and 5 investigate the optimal disclosure policy for a principal who maximizes aggregate effort: in Sect. 4 the principal can only commit to either fully disclosing or fully concealing contestants' types, and in Sect. 5 the principal can commit to disclosing contestants' types contingently on the realization of types. Section 6 discusses the results. Appendix A contains the proofs and Appendix B extensions.

\section{Related literature}

The most closely related literature is that on how information affects efforts in Tullock contests (e.g., Hurley and Shogren 1998a; Hurely and Shogren 1998b; Denter et al. 2011; Epstein and Mealem 2013; Heijnen and Schoonbeek 2016; Zhang and Zhou 2016; Chen et al. 2017, 2018b). These papers - in contrast to ours — assume that the type of one contestant is common knowledge and that of the other is private information. In other words, the private information the principal may disclose is one-sided among the two contestants. We believe that a disclosure policy equally capable of disclosing each contestant's type is a sensible assumption in the applications discussed in the Introduction. From a more technical perspective, the prevailing assumption of one-sided private information contest shuts down the higher-order reasoning behind the $p$-Effects which drives the results of the present paper. Perhaps one reason for the popularity of the one-dimensional private information approach is its tractability, in that it admits a closed-form solution for equilibrium efforts, contrary to our context. ${ }^{4}$ In fact, in the words of Zhang and Zhou (2016), "It is natural to ask what happens if both contestants possess private information. Several technical challenges emerge accordingly, $[\ldots]$ a characterization of the equilibrium is usually not obtainable." We sidestep the lack of a closed-form solution by showing that, in Tullock contests, equilibrium efforts always satisfy a property that depends on contestants' beliefs about the rival's type (see (6) in Appendix A), and that this property suffices to fully characterize the optimal disclosure policy. Furthermore, this novel property happens to generalize a well-known wisdom of complete information Tullock contests-namely the ratio

4 Even if $r=1$ in (1), a contest does not admit a closed-form solution for equilibrium efforts in our fully-private information setting unless $p \in\{0,1 / 2,1\}$. The case $p=1 / 2$ is analyzed in Malueg and Yates (2004). 
of types equals the ratio of efforts-to Tullock contests with a variety of contestants' beliefs about the rival's type.

The literature has proposed ways that sidestep the lack of a closed-form solution for the equilibrium efforts of private information Tullock contests: among others, a modified contest success function (see Wasser 2013), a binary effort space (see Dubey 2013), or numerical simulations (see Hurley and Shogren 1998a; Wasser 2013). In particular, Wasser (2013) compared full disclosure and full concealment through numerical simulations, while in Theorem 1 we formally prove his numerical result under binary distribution of types; however, not having an analytical result impeded him from explaining the reason why the probability of high- and low-types is what drives the optimal disclosure policy. The key role of the probability of high- and lowtypes is one of our main contributions, and we show how it extends to some non-binary distributions (see, Appendix B.3).

The present paper is, to the best of my knowledge, the first to study type-contingent information disclosure in contests with two-sided asymmetric information. Following the present paper, Chen et al. (2018a) study the joint optimal design of timing and information disclosure in Tullock contests, and Lu et al. (2018) study the optimal information disclosure in all-pay auctions, where an arbitrarily higher effort secures victory. ${ }^{5}$ In particular, Lu et al. (2018) extend the present analysis to all-pay auctions. Their work considerably contributes to the literature in that they provide a full characterization of the equilibrium bidding function in all-pay auctions under a regime of contingent information disclosure, where Siegel's (2014) approach is not applicable. In contrast to our Tullock success function, the all-pay auction yields payoffs which are not quasiconcave, hence leading to a nondegenerate mixed strategy equilibrium even in the complete information case. This generates structural differences between the two settings which make the results hardly comparable. For instance, in Lu et al.'s (2018) equilibrium participation depends on beliefs and distribution of types. Furthermore, the elegant characterization of the $p$-Effects is possible thanks to the strategic complementarity (substitutability) of the high- (low-) type's effort around the equilibrium, which we find in our setup with Tullock contest success function with exponent $r \leq 1 .^{6}$

Other branches of the literature are related to the present paper. First, the literature on the disclosure of contestants' dynamic performance (e.g., Aoyagi 2010; Goltsman and Mukherjee 2011) or of the number of contestants (e.g., Myerson and Wärneryd 2006; Lim and Matros 2009; Fu et al. 2011). Second, the literature on contestants' incentive to acquire information (e.g., Yildirim 2005; Denter et al. 2011). In our model it is the principal, rather than the contestants, who has control over the information that contestants acquire. Third, the literature on contestants' incentive to disclose their private information. While players have no incentive to do so in all-pay auctions (e.g., Kovenock et al. 2015), in Tullock contests players may want to disclose their information (Wu and Zheng 2017). Fourth, and finally, the Bayesian persuasion literature

\footnotetext{
5 An earlier version of the present paper is Serena (2016), which Lu et al. (2018) cite. The earliest version is Serena (2014); see the reference in Vázquez Sedano (2015).

6 Beside Lu et al.'s (2018) extension of our results to all-pay auctions, the comparison between expected aggregate effort under full disclosure or full concealment is in Fu et al. (2014) and Kovenock et al. (2015), who find that full concealment dominates full disclosure.
} 
(see Kamenica and Gentzkow 2011, henceforth KG), where a sender commits to a disclosure policy on a stochastic state of the world before its realization. In this class of models, it is not a novelty to find optimality of contingent information disclosure, like in our setting; in particular, it is optimal for a sender (i.e., a prosecutor in $\mathrm{KG}$, and the principal here) to disclose to a receiver (i.e., a judge in $\mathrm{KG}$, and the set of two contestants here) only the realization which is most favorable to the sender (i.e., the defendant being guilty in $\mathrm{KG}$, and both contestants being high-types here) and to pool the other signals in a strategic way (i.e., randomizing between innocent and guilty signals in $\mathrm{KG}$, and concealing here). The disclosure policy space is more general under the Bayesian persuasion approach than in our setting. Nevertheless, our disclosure policy space is simpler to implement, in that it involves no stochastic messages, and thus it requires a weaker commitment condition than Bayesian persuasion. Furthermore, our disclosure policy space is not affected by the technical challenges of applying Bayesian persuasion to analyze the disclosure of both contestants' types. (Three such technical challenges are discussed by Zhang and Zhou 2016; Section 4).

\section{A model of a contest}

The contest technology. Two contestants, indexed by $i=1,2$, compete for a prize of value $V>0$ by exerting effort $e_{i} \geq 0$. Each contestant has a probability of winning a prize equal to ${ }^{7}$

$$
p_{i}\left(e_{i}, e_{j}\right)=\left\{\begin{array}{cl}
\frac{e_{i}^{r}}{e_{i}^{r}+e_{j}^{r}} & \text { if } e_{i}+e_{j}>0 \\
\frac{1}{2} & \text { if } e_{i}+e_{j}=0
\end{array}\right.
$$

with $i, j=1,2, j \neq i$, and $0<r \leq 1$. When at least one player exerts strictly positive effort, (1) uniquely satisfies a set of appealing axioms (see Skaperdas 1996).

The contestants' payoff. Contestants are risk-neutral. The cost of effort is linear, and contestant $i$ is of type $\theta_{i}$, which determines her marginal cost of effort. In particular, the payoff of a contestant of type $\theta_{i}$ when she exerts effort $e_{i}$ and her rival exerts effort $e_{j}$ is

$$
p_{i}\left(e_{i}, e_{j}\right) V-\frac{e_{i}}{\theta_{i}}
$$

Contestant's type $\theta_{i}$ is an independent draw from the commonly-known prior, ${ }^{8}$

$$
\theta_{i}= \begin{cases}h & \text { with probability } p \\ l & \text { with probability } 1-p\end{cases}
$$

with $p \in[0,1]$ and $h>l>0$. Thus, being a high-type rather than a low-type brings about a lower marginal cost of effort.

\footnotetext{
7 In Appendix B.4 we briefly discuss a more general contest technology.

8 In Appendix B.3 we discuss the case of a continuum of types.
} 
Table 1 Possible realizations of contestants' types, with corresponding probability and aggregate equilibrium effort under $\mathcal{P}=\mathcal{D}$ and $\mathcal{P}=\mathcal{C}$

\begin{tabular}{llll}
\hline Realizations of $\left\{\theta_{1}, \theta_{2}\right\}$ & Prob. & Aggregate effort if $\mathcal{D}$ & Aggregate effort if $\mathcal{C}$ \\
\hline$\{h, h\}$ & $p^{2}$ & $2 e_{h h}$ & $2 e_{h}$ \\
$\{l, l\}$ & $(1-p)^{2}$ & $2 e_{l l}$ & $2 e_{l}$ \\
$\{h, l\}$ or $\{l, h\}$ & $2 p(1-p)$ & $e_{h l}+e_{l h}$ & $e_{h}+e_{l}$ \\
\hline
\end{tabular}

The timing of the game. First, before types are realized, the principal commits to a verifiable and costless disclosure policy $\mathcal{P}$, which is observed by the contestants. Then, types are realized, each contestant $i$ learns $\theta_{i}$ and may or may not be informed by the principal of $\theta_{j}$, according to $\mathcal{P}$. Finally, contestants simultaneously choose efforts.

The principal and the disclosure policy. The principal maximizes the expected aggregate effort when choosing the disclosure policy $\mathcal{P} .{ }^{9}$ In Sect. 4, the principal chooses between two extreme disclosure policies on types: disclosure $\mathcal{P}=\mathcal{D}$ (that is, contestants are informed of their rival's type), and concealment $\mathcal{P}=\mathcal{C}$ (that is, contestants are not informed and contestants' posterior belief about their rival's types equals the prior (2)). In Sect. 5 we enlarge the space of $\mathcal{P}$ 's: the principal commits to disclosing or concealing contestants' types contingently on type realizations. ${ }^{10}$ Each $\mathcal{P}$ induces a system of beliefs for the contestants, and each system of beliefs induces an expected level of aggregate effort. We discuss the $\mathcal{P}$ which maximizes aggregate effort in the main text, while intermediary results on how systems of beliefs affect aggregate effort appear in "Appendix A".

Equilibrium. Since contestants are ex-ante symmetric, it is natural to focus on typesymmetric equilibria; that is, contestants of the same type follow the same equilibrium strategy. The existence of a unique equilibrium has already been proved in our setting (see Einy et al. 2015, and Ewerhart and Quartieri 2019). Our results in the main text are valid for both interior and corner equilibria; formally, we assume interior equilibrium throughout the paper, and at the end of "Appendix A" we build on our findings to explain why considering corner equilibria does not affect our results.

\section{Optimal disclosure policy: disclose or conceal?}

If the principal chooses between committing to full disclosure $(\mathcal{P}=\mathcal{D})$ or full concealment $(\mathcal{P}=\mathcal{C})$ only, which one yields the greatest expected aggregate effort? When committing to a $\mathcal{P}$, the principal does not know whether the contest will be between two high-types, two low-types, or a high-type and a low-type. Table 1 provides a summary of the possible realizations of types, the corresponding probabilities, and the aggregate equilibrium effort (i.e., the principal's payoff). The notation is as follows: the first subindex in $e$ is the type of the contestant exerting effort, and the

\footnotetext{
9 In Appendix B.2 we discuss alternative objective functions for the principal.

10 In Appendix B.1 we discuss the optimal disclosure policy if the principal does not have commitment power, so that the disclosure policy is de facto chosen ex-post.
} 


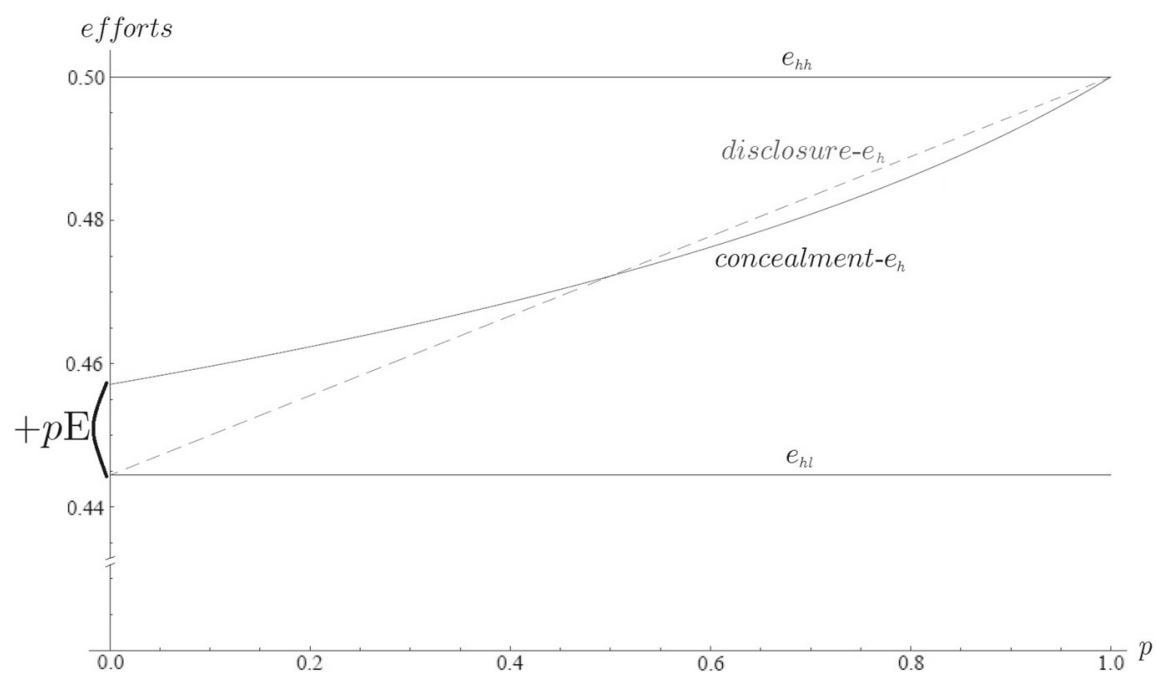

Fig. 1 High-type's equilibrium efforts as functions of $p$ assuming $r=1, V=1, l=1$ and $h=2$. The lines $e_{h h}$ and $e_{h l}$ are her two efforts under $\mathcal{D}$. The dashed line disclosure $-e_{h}$ is thus the principal's expectation of a high-type's effort under $\mathcal{D}$. The line concealment- $e_{h}$ is the effort of a high-type under $\mathcal{C}$. The segment called $+p \mathrm{E}$ is the positive $p$-Effect, which is depicted at $p \rightarrow 0$

second subindex is the type of her rival, in case she is told (that is, under $\mathcal{D}$ ). Thus, for instance, $e_{h l}$ is the equilibrium effort of a high competing against a low (under $\mathcal{D}$ ), and $e_{h}$ is the equilibrium effort of a high who does not know her rival (under $\mathcal{C}$ ).

If the contest is even- $\{h, h\}$ or $\{l, l\}-$, then $\mathcal{D}$ maximizes aggregate effort because it prevents contestants from thinking that they are competing in an uneven contest (i.e., $2 e_{h h} \geq 2 e_{h}$ and $\left.2 e_{l l} \geq 2 e_{l}\right)$. If instead the contest is uneven- $\{h, l\}$ or $\{l, h\}-$, then $\mathcal{C}$ maximizes aggregate effort because it prevents discouragement. Therefore, finding the optimal $\mathcal{P}$ boils down to the ex-ante trade-off between the benefits of $\mathcal{D}$ if the contest is even and the benefits of $\mathcal{C}$ if the contest is uneven. In what follows we gradually build the intuition that will eventually lead to Theorem 1 using the graphical support of Figs. 1 and 2.

In Fig. 1 we focus on the effort of a high-type. ${ }^{11}$ The horizontal lines are her two possible equilibrium efforts under disclosure-against another high-type or against a low-type-, where $p$ does not affect efforts and $e_{h h}>e_{h l}$ because evenness of types stimulate efforts. We call disclosure- $e_{h}$ the effort that the principal expects from the high-type under disclosure, which equals $p e_{h h}+(1-p) e_{h l}$. Thus, the disclosure- $e_{h}$ is a straight line in $p$ going from $e_{h l}$ to $e_{h h}$, as depicted. We call concealment- $e_{h}$ the equilibrium effort of a high-type under concealment. Ranking concealment- $e_{h}$ and disclosure- $e_{h}$ (and concealment- $e_{l}$ and disclosure- $e_{l}$ in Fig. 2) unveils the optimal disclosure policy. The concealment- $e_{h}$ is increasing in $p$ because $p$ increases the probability of an even contest. When $p \rightarrow 0$, the high-type believes she is up against

11 Since a closed-form solution for equilibrium efforts does not exist, figures are created using numerical simulations on the system of FOCs (see (4) and (5) in "Appendix A") with $r=1, V=1, l=1$ and $h=2$. This parametrization does not affect the qualitative features of those figures. 


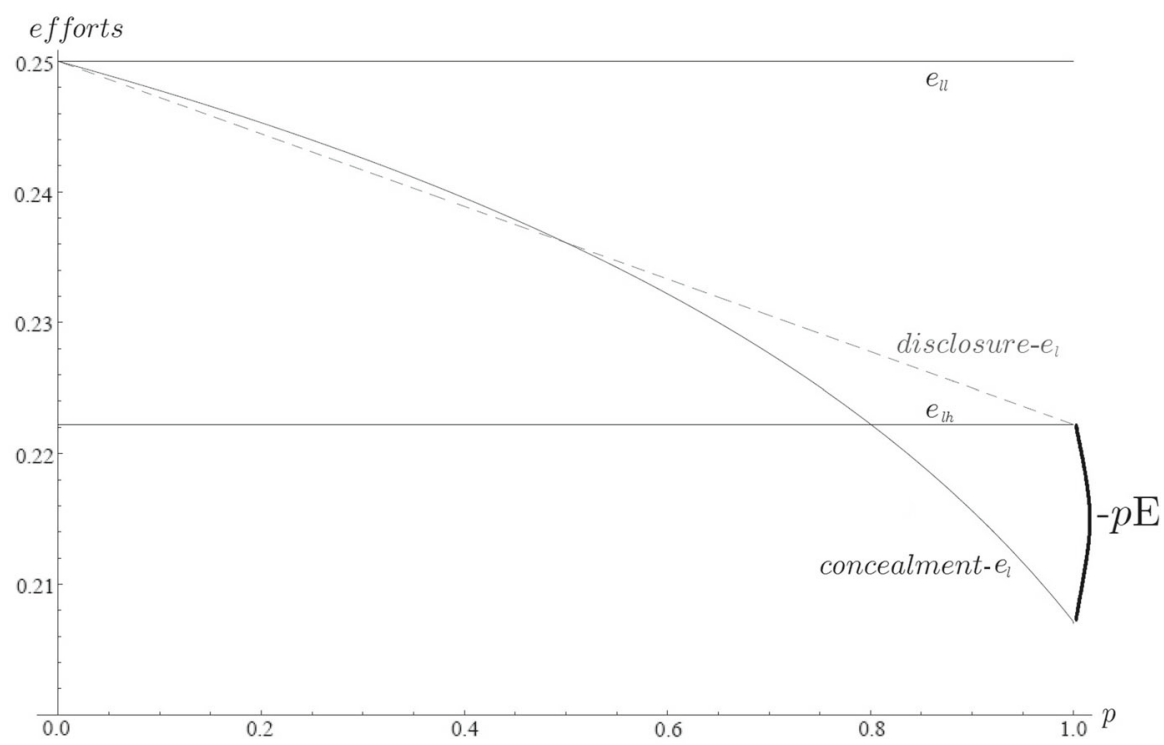

Fig. 2 Low-type's equilibrium efforts as functions of $p$ assuming $r=1, V=1, l=1$ and $h=2$. The lines $e_{l l}$ and $e_{l h}$ are her two efforts under $\mathcal{D}$. The dashed line disclosure $-e_{l}$ is thus the principal's expectation of a low-type's effort under $\mathcal{D}$. The line concealment- $e_{l}$ is the effort of a low-type under $\mathcal{C}$. The segment called - $p \mathrm{E}$ is the negative $p$-Effect, which is depicted at $p \rightarrow 1$

a low-type who believes she is up against another low-type who also believes she is up against another low-type, and so on. In other words, the high-type expects an uneven contest against a rival who expects an even contest with commonly known types. This implies that concealment- $e_{h}$ is greater than the disclosure- $e_{h}$ (under disclosure, the contestants' unevenness is commonly known). ${ }^{12}$ This is what we called $+p \mathrm{E}$. The more $p$ increases in the interval $p \in(0,0.5)$, the less likely there are situations where the high-type's and the low-type's beliefs about the evenness of the contest sharply differ (as when $p \rightarrow 0$ ), and this is what makes the $+p \mathrm{E}$ gradually disappear and the concealment- $e_{h}$ converge to the disclosure- $e_{h}$. When $p=0.5$, contestants believe they are taking part in an even or uneven contest with equal probability, and they know that their rival, regardless of types, has the same beliefs: that is, the two opposing forces - namely being discouraged (by an uneven contest) and stimulated (by an even contest)—balance out, and thus the higher-order reasoning "does not distort" efforts. Additionally, when $p \in(0.5,1)$, the concealment $-e_{h}$ is below the disclosure- $e_{h}$. The reason is that a high-type believes she is more likely up against another high-type who also believes she is more likely up against another high-type, and so on. However, the high-type knows that if she happens to be against a low-type (i.e., the less likely contingency), her low-type rival will be discouraged (as she believes she is up against a high-type who believes she is up against another high-type, and so on; shortly, the $-p \mathrm{E}$ ), thus exerting low effort; the possibility of being with low probability $1-p$

12 Note that when $p=0$, both concealment- $e_{h}$ and disclosure- $e_{h}$ do not matter because a high-type does not exist. Nevertheless, we start our explanation with the limiting case $p \rightarrow 0$ because this is where the $p$-Effect can be immediately grasped. 


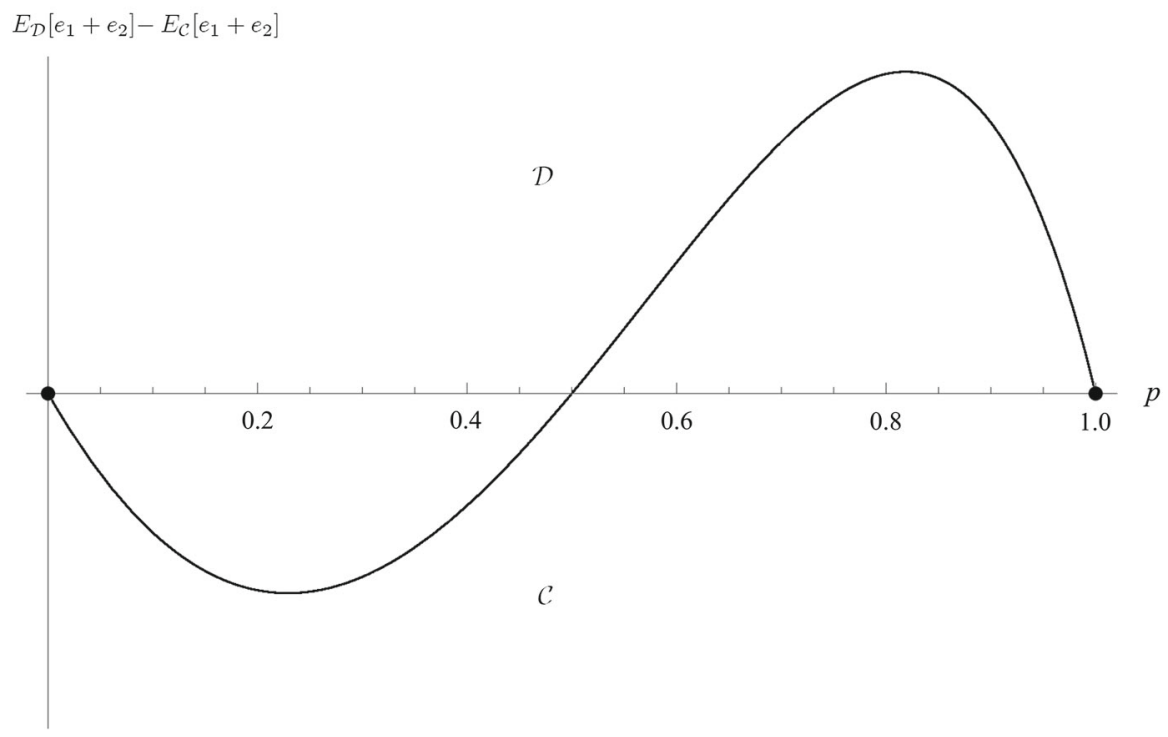

Fig. $3 E_{\mathcal{D}}\left[e_{1}+e_{2}\right]-E_{\mathcal{C}}\left[e_{1}+e_{2}\right]$ : Difference between the expected aggregate effort under $\mathcal{D}$ and under $\mathcal{C}$ as a function of $p$, assuming $r=1, V=1, l=1$ and $h=2$

against a discouraged low-type decreases the effort of the high-type with respect to a disclosure setting, where such possibility is clearly not present.

In Fig. 2 the same exercise is carried out for a low-type, with symmetric results. We briefly repeat the reasoning we made above for a high-type. When $p \rightarrow 1$, the disclosure- $e_{l}$ is greater than the concealment- $e_{l}$ because a low-type believes she is up against a high-type who believes she is up against another high-type, and thus the low-type exerts less effort than $e_{l h}$ (i.e., a low-type is discouraged by $-p \mathrm{E}$ ). When $p=0.5$, the $-p \mathrm{E}$ disappears and the disclosure- $e_{l}$ equals the concealment- $e_{l}$, while when $p \in(0,0.5)$ the concealment- $e_{l}$ is above the disclosure- $e_{l}$.

Therefore, all forces affecting the high-type and low-type's efforts point at the same conclusion: for $p \in[0,0.5]$ both concealment- $e_{h}$ and concealment- $e_{l}$ are greater than the disclosure ones, while for $p \in[0.5,1]$ they are both lower. Thus, if $p \in[0,0.5]$ concealment is the optimal policy, and if $p \in[0.5,1]$ disclosure is the optimal policy. These results lead to the optimal $\mathcal{P}$ of Theorem 1, which is depicted in Fig. 3. ${ }^{13}$

Theorem 1 Under full disclosure or full concealment only, the optimal disclosure policy for the principal is to commit to full disclosure (full concealment) if high-types are more (less) likely than low-types; that is, if $p \in[0.5,1](p \in[0,0.5])$. The principal is indifferent between full concealment and full disclosure if and only if the prior is degenerate, i.e., $p \in\{0,1\}$, or symmetric, i.e., $p=0.5$.

\footnotetext{
13 Although parameters $h$ and $l$ do not affect the principal's optimal disclosure policy, the absolute value of the difference between aggregate effort under $\mathcal{D}$ and under $\mathcal{C}$ increases in the ratio $h / l$. In other words, the vertical scale of Fig. 3 shrinks for a reduction of $h / l$, all the way down to the case of $h=l$, that is, a flat horizontal line depicting indifference between any $\mathcal{P}$ for all values of $p$. The same holds for $r$ and $V$, which do not affect the principal's optimal disclosure policy, but scale up or down efforts in each $\mathcal{P}$. The independence of the optimal $\mathcal{P}$ on $h, l, r$ and $V$ also holds in Sect. 5.
} 
Despite Theorem 1 answering a very natural question, a plausible reason why the present paper is the first to provide an answer is the well-known lack of a closed-form solution for efforts under two-sided private information Tullock contests. In the proof of Theorem 1 (and later on of Theorem 2), we sidestep the lack of a closed-form solution by showing that equilibrium efforts always satisfy a property that depends on contestants' beliefs about the rival's type (in "Appendix A", see (6) for Theorem 1, and the corresponding (22)-(23) for Theorem 2), and that this property suffices to fully characterize the optimal disclosure policy.

Theorem 1 is the first of the two main results of the paper. We challenge this result with five extensions in "Appendix B". First, we consider ex-post rather than ex-ante disclosure; we find that the equilibrium efforts are those of full disclosure. Second, we consider objectives other than the maximization of aggregate effort; we obtain a similar optimal disclosure policy if the principal maximizes the expected winning effort, and the opposite optimal disclosure policy to that of Theorem 1 when the principal minimizes aggregate effort or maximizes the probability of a high-type winner. Third, we consider the optimal disclosure policy with two tractable cases of continuum-type distribution drawn from the literature; we find that the link between the relative likelihood of high- and low-types and optimal disclosure policy goes in the same direction as that characterized in Theorem 1. Fourth, we spell out the technical difficulties of deriving the optimal disclosure policy under a contest success function more general than (1).

\section{Optimal contingent disclosure policy}

In Sect. 4, the principal could only commit to fully disclosing or fully concealing contestants' types. Can the principal do any better using a partial disclosure policy? This section shows that the answer is positive, regardless of $p$, in a specific family of partial disclosure policies, where the principal commits to disclosing or concealing contestants' types contingently on the realization of types. The principal needs a stronger commitment power in order to implement such a contingent information disclosure; online contests are a setting where such a broadening of the disclosure policy space is easily implementable.

$\mathcal{P}$ is now a vector of three binary variables, each taking the value $\mathcal{D}$ or $\mathcal{C}$, where the first (respectively, second and third) element corresponds to the disclosure choice in case of realization $\{h, h\}$ (respectively, $\{h, l\}$ and $\{l, l\}$ ). Realizations $\{h, l\}$ and $\{l, h\}$ are equivalent for the principal, who hence has no incentive to choose different disclosure policies to $\{h, l\}$ and $\{l, h\}$. Although the set of $\mathcal{P}$ analyzed in this section formally nests the two extreme cases analyzed in Sect. $4-\mathcal{P}=\{\mathcal{C}, \mathcal{C}, \mathcal{C}\}$ corresponds to full concealment, and $\mathcal{P}=\{\mathcal{D}, \mathcal{D}, \mathcal{D}\}$ to full disclosure-, we keep the two sections separate because: i) the comparison of public and private information contests is of self-interest, and ii) the more sophisticated disclosure policy in this section might not be implementable in some real-life situations. As in Sect. $4, \mathcal{P}$ is publicly announced ex-ante by the principal who commits to it, and no randomization is allowed.

The result of this section is that $\mathcal{P}=\{\mathcal{D}, \mathcal{C}, \mathcal{C}\}$ is optimal for all $p$ 's - that is, disclosure only when both contestants are high-types yields an expected aggregate 
effort greater than that of full disclosure, full concealment, and any other contingent disclosure policy.

Theorem 2 Under contingent information disclosure, the optimal disclosure policy for the principal is to commit to disclosing contestants' types only when the realization is $\{h, h\}$, if $p \in(0,1)$. That is, $\mathcal{P}=\{\mathcal{D}, \mathcal{C}, \mathcal{C}\}$. If $p \in\{0,1\}$, the principal is indifferent between any disclosure policies.

The intuition behind Theorem 2 is as follows. First, consider how $\mathcal{P}=\{\mathcal{D}, \mathcal{C}, \mathcal{C}\}$ affects contestants' beliefs. A high-type who is not told her rival's type infers she must be up against a low-type. Thus, (A) a high-type always knows her rival's type, and (B) a low-type never knows her rival's type. More importantly, (A) and (B) are common knowledge; in particular, low-types know (A) and high-types know (B). Therefore, such a $\mathcal{P}$ allows the principal, regardless of $p$, to both:

- avoid the losses due to-pE. - $p \mathrm{E}$ is an effort loss that occurs when a low-type is up against a high-type who is not aware of being up against a low-type. This situation does not occur under such a $\mathcal{P}$ because low-types know (A), and thus even if a low-type is up against a high-type, the low-type knows that the high-type is aware of being up against a low-type.

- benefit from $+\mathbf{p E} .+p \mathrm{E}$ is an effort gain that occurs when a high-type is up against a low-type who is not aware of being up against a high-type. This situation does occur under such a $\mathcal{P}$ because high-types know (B), and thus a high-type knows that, when up against a low-type, this low-type will not be aware of being up against a high-type.

Therefore, the intuition behind Theorem 2 relies on $+p \mathrm{E}$ and $-p \mathrm{E}$, as the one behind Theorem 1. Yet, the optimal disclosure policy of Theorem 1 depends on $p$ whereas the one of Theorem 2 does not. The reason is that, if only full disclosure or full concealment are possible, the principal's priority is either to avoid the losses due to - $p \mathrm{E}$ (and thus disclose) when $p$ is high or to make the most out of the $+p \mathrm{E}$ (and thus conceal) when $p$ is low, whereas if the disclosure policy space admits contingent disclosures, the principal can simultaneously achieve both goals for all $p$ 's.

While the worst (or effort-minimizing) disclosure policy of Sect. 4 immediately follows from Theorem 1, Theorem 2 does not characterize the worst contingent disclosure policy. For the sake of completeness, we characterize the worst disclosure policy when contingent information disclosure is possible.

Proposition 3 Under contingent information disclosure and $p \in(0,1)$, the worst disclosure policy for the principal is to commit to disclosing contestants' types only when the realization is $\{l, l\}$. That is, $\mathcal{P}=\{\mathcal{C}, \mathcal{C}, \mathcal{D}\}$.

The intuition behind Proposition 3 is the mirror image of that of Theorem 2; namely by disclosing only realization $\{l, l\}$ the principal suffers the losses due to $-p \mathrm{E}$ and at the same time she does not obtain the benefits of $+p$ E. Additionally, Proposition 3 , together with Theorem 2 and Theorem 1, yields the full ranking of contingent disclosure policies;

Corollary 4 Consider contingent information disclosure and $p \in(0,1)$. If $p \in$ $(0,0.5)$, the full ranking of policies is

$$
\{\mathcal{D}, \mathcal{C}, \mathcal{C}\} \succ\{\mathcal{C}, \mathcal{C}, \mathcal{C}\} \succ\{\mathcal{D}, \mathcal{D}, \mathcal{D}\} \succ\{\mathcal{C}, \mathcal{C}, \mathcal{D}\}
$$


If $p \in(0.5,1)$, the full ranking of policies is

$$
\{\mathcal{D}, \mathcal{C}, \mathcal{C}\} \succ\{\mathcal{D}, \mathcal{D}, \mathcal{D}\} \succ\{\mathcal{C}, \mathcal{C}, \mathcal{C}\} \succ\{\mathcal{C}, \mathcal{C}, \mathcal{D}\}
$$

If $p=0.5$, the full ranking of policies is

$$
\{\mathcal{D}, \mathcal{C}, \mathcal{C}\} \succ\{\mathcal{D}, \mathcal{D}, \mathcal{D}\} \sim\{\mathcal{C}, \mathcal{C}, \mathcal{C}\} \succ\{\mathcal{C}, \mathcal{C}, \mathcal{D}\}
$$

\section{Discussion}

We have studied a model of contest where contestants are privately informed of their types and the principal can commit to publicly disclosing contestants' types. We have characterized the optimal disclosure policy when the principal maximizes the expected aggregate effort of the contestants. In particular, full disclosure extracts more (less) expected aggregate effort than full concealment if high-types are more (less) likely than low-types. Moreover, if contingent information disclosure is possible, the optimal disclosure policy is to commit to disclosing contestants' types only when the realization of types which is most favorable for the principal - i.e., contestants are all high-typesand to concealing the rest.

We have shown that the effect of the disclosure policy on the contestants' higherorder reasoning (which we called $+p \mathrm{E}$ and $-p \mathrm{E}$ ) is of first-order importance. In fact, we find that $+p \mathrm{E}$ and $-p \mathrm{E}$ are the only forces that affect the efforts of the high-type and low-type with opposite sign when high-types are more and less likely than lowtypes. This asymmetry is the by-product of the fact that, around the equilibrium, a high-type's strategy shows strategic complementarity and a low-type's strategy shows strategic substitutability. By no means do we attempt to make a statement that optimal disclosure policies depend in general only on the $p$-Effects. Rather, two-player Tullock contests are a suitable environment for singling out and stressing the relevance of the $p$-Effects which, although not convoluted, have not received attention in earlier papers, to the best of our knowledge.

Three shortcomings of our analysis pinpoint room for future research. First, we ignored contestants' selection. It may be effort-improving to endow the model with an entry fee capable of sifting out weak applicants. Second, we abstracted away from contestants' participation decision. The announced disclosure policy itself may affect contestants' willingness to participate, and we conjecture that concealment deters the low-types from entering, and that this might be beneficial in terms of expected aggregate effort. Third, the disclosure of information about other contestants that would otherwise be unknown to contestants has other consequences in addition to the one considered in the present paper. In fact, disclosure may also trigger communication among contestants, affect the external visibility of contestants, and create animosity among contestants. We abstracted away from these effects and focused here on the direct effects on efforts of making contestants' types publicly available to other contestants.

Funding Open Access funding enabled and organized by Projekt DEAL. 
Open Access This article is licensed under a Creative Commons Attribution 4.0 International License, which permits use, sharing, adaptation, distribution and reproduction in any medium or format, as long as you give appropriate credit to the original author(s) and the source, provide a link to the Creative Commons licence, and indicate if changes were made. The images or other third party material in this article are included in the article's Creative Commons licence, unless indicated otherwise in a credit line to the material. If material is not included in the article's Creative Commons licence and your intended use is not permitted by statutory regulation or exceeds the permitted use, you will need to obtain permission directly from the copyright holder. To view a copy of this licence, visit http://creativecommons.org/licenses/by/4.0/.

\section{Appendix A: Proofs}

In this Appendix, we prove Theorem 1 and Theorem 2 (together with the full ranking of policies of Proposition 3 and Corollary 4) by first deriving some equilibrium properties as "Preliminaries", and second building on those preliminaries to prove the main results in a number of "Steps".

\section{Proof of Theorem 1}

We adopt the compact notation $\pi^{\mathcal{D}-\mathcal{C}}$ for $E_{\mathcal{D}}\left[e_{1}+e_{2}\right]-E_{\mathcal{C}}\left[e_{1}+e_{2}\right]$; that is, the difference in the expected sum of efforts under $\mathcal{D}$ and under $\mathcal{C}$, which is depicted in Fig. 3 as a function of $p$.

First, we spell out some Preliminaries. Second, we prove Theorem 1 in three steps. Step 1: $\pi^{\mathcal{D}-\mathcal{C}}=0$ iff $p \in\{0,0.5,1\}$. Step 2: The derivative of $\pi^{\mathcal{D}-\mathcal{C}}$ with respect to $p$ at $p=0.5$ is strictly positive. Step 3: $\pi^{\mathcal{D}-\mathcal{C}}$ is continuous in $p$. These three results together lead to the sign of $\pi^{\mathcal{D}-\mathcal{C}}$ as in Fig. 3, hence characterizing the optimal disclosure policy of Theorem 1 without the need for closed-form equilibrium efforts under $\mathcal{C}$.

Preliminaries The existence and uniqueness of equilibrium in pure strategies is already present in the literature. ${ }^{14}$

Under $\mathcal{D}$, as known in the literature (e.g., Nti 1999), equilibrium efforts are ${ }^{15}$

$$
e_{h h}=\frac{r h}{4} V, \quad e_{h l}=\frac{r h^{r+1} l^{r}}{\left(h^{r}+l^{r}\right)^{2}} V, \quad e_{l h}=\frac{r l^{r+1} h^{r}}{\left(h^{r}+l^{r}\right)^{2}} V, \quad e_{l l}=\frac{r l}{4} V .
$$

Under $\mathcal{C}$, the system of FOCs has two unknowns $\left(e_{h}\right.$ and $\left.e_{l}\right)$, and reads

$$
\begin{gathered}
\text { (FOC of } h \text {-type) }: \quad p \frac{r}{4 e_{h}} V+(1-p) \frac{r e_{h}^{r-1} e_{l}^{r}}{\left[e_{h}^{r}+e_{l}^{r}\right]^{2}} V=\frac{1}{h}, \\
\left(\text { FOC of } l \text {-type) }: \quad(1-p) \frac{r}{4 e_{l}} V+p \frac{r e_{l}^{r-1} e_{h}^{r}}{\left[e_{h}^{r}+e_{l}^{r}\right]^{2}} V=\frac{1}{l} .\right.
\end{gathered}
$$

\footnotetext{
${ }^{14}$ See Szidarovszky and Okuguchi (1997) for the existence and uniqueness under complete information. Under incomplete information, Einy et al. (2015) and Ewerhart and Quartieri (2019) prove existence and uniqueness in a setting that nests our model.

15 The notation for the subindexes of equilibrium efforts is the one introduced at the beginning of Sect. 4.
} 
The FOCs are necessary and sufficient to characterize the best reply, which is continuously differentiable and bounded. ${ }^{16}$ To lighten the notation we omit the dependencies of $e_{h}$ and $e_{l}$ on $p, h, l, r$ and $V$. A type-symmetric equilibrium is a pair $\left(e_{h}, e_{l}\right)$ satisfying (4) and (5).

Finally, isolate the second addends of the left-hand sides in (4) and (5), consider their ratio,

$$
\begin{aligned}
\frac{(1-p) r e_{h}^{r-1} e_{l}^{r}}{p r e_{l}^{r-1} e_{h}^{r}} & =\frac{\frac{1}{h}-p \frac{r}{4 e_{h}} V}{\frac{1}{l}-(1-p) \frac{r}{4 e_{l}} V} \\
& \Longleftrightarrow \frac{(1-p) e_{l}}{p e_{h}}=\frac{\frac{1}{h}-p \frac{r}{4 e_{h}} V}{\frac{1}{l}-(1-p) \frac{r}{4 e_{l}} V}
\end{aligned}
$$

cross-multiply,

$$
\begin{aligned}
\frac{(1-p) e_{l}}{l}-(1-p)^{2} \frac{r}{4} V & =\frac{p e_{h}}{h}-p^{2} \frac{r}{4} V \\
& \Longleftrightarrow \frac{4}{r V} \frac{(1-p) e_{l}}{l}=\frac{4}{r V} \frac{p e_{h}}{h}+(1-2 p),
\end{aligned}
$$

and finally use the expressions of $e_{h h}$ and $e_{l l}$ in (3), in order to derive the following key relation between equilibrium efforts under $\mathcal{D}$ and under $\mathcal{C}$,

$$
\frac{e_{l}}{e_{l l}}(1-p)=\frac{e_{h}}{e_{h h}} p+(1-2 p) .
$$

Using the above preliminaries (i.e., (3)-(6)), we are ready to proceed with the three main steps needed to prove Theorem 1 .

Step 1. We show that $\pi^{\mathcal{D}-\mathcal{C}}=0$ iff $p \in\{0,0.5,1\}$. First, we analyze when $\pi^{\mathcal{D}-\mathcal{C}}$ takes the value 0 .

$$
\pi^{\mathcal{D}-\mathcal{C}}=p^{2}\left[2 e_{h h}-2 e_{h}\right]+2 p(1-p)\left[e_{h l}+e_{l h}-e_{h}-e_{l}\right]+(1-p)^{2}\left[2 e_{l l}-2 e_{l}\right]=0,
$$

i.e.,

$$
p^{2} e_{h h}+(1-p)^{2} e_{l l}+p(1-p)\left[e_{h l}+e_{l h}\right]-p e_{h}-(1-p) e_{l}=0
$$

If $p=0$ or $p=1$, by $e_{l}=e_{l l}$ and $e_{h}=e_{h h}$ respectively, (7) holds and $\pi^{\mathcal{D}-\mathcal{C}}=0$. Thus, from now on we can focus on $p \in(0,1)$.

16 See Yildirim (2005) and Morgan and Várdy (2007). 
Substitution of (3) and (6) into (7) yields

$$
\begin{aligned}
& p^{2} \frac{r h}{4} V+(1-p)^{2} \frac{r l}{4} V+p(1-p) \frac{r(h+l) h^{r} l^{r}}{\left(h^{r}+l^{r}\right)^{2}} V-p \frac{h+l}{h} e_{h}-\frac{1-2 p}{4} r l V=0, \\
& p r \frac{h+l}{4} V+(1-p) \frac{r(h+l) h^{r} l^{r}}{\left(h^{r}+l^{r}\right)^{2}} V=\frac{h+l}{h} e_{h}, \\
& p e_{h h}+(1-p) e_{h l}=e_{h} .
\end{aligned}
$$

Hence, (8) is a condition for the indifference between $\mathcal{D}$ and $\mathcal{C}$ written in terms of the efforts exerted by the high-type only. This condition coincides with equality of concealment $-e_{h}$ and disclosure- $e_{h}$ (see Fig. 1). With a similar procedure used to find (8) - that is, by substituting (6) into $e_{h}$ rather than into $e_{l}$-we can obtain the value of $e_{l}$ for which the administrator is indifferent between $\mathcal{D}$ and $\mathcal{C}$, which symmetrically to $(8)$ is

$$
(1-p) e_{l l}+p e_{l h}=e_{l}
$$

This condition coincides with equality of concealment- $e_{l}$ and disclosure- $e_{l}$ (see Fig. 2). We plug (8) and (9) into (4) and see if any $p \in(0,1)$ solves the resulting equationthus, yielding $\pi^{\mathcal{D}-\mathcal{C}}=0 .{ }^{17}$

First, use (3) to rewrite the indifference conditions (8) and (9) for $e_{l}$ and $e_{h}$ as

$$
\begin{aligned}
& e_{h}=r h \frac{p\left(h^{r}+l^{r}\right)^{2}+4(1-p) h^{r} l^{r}}{4\left(h^{r}+l^{r}\right)^{2}} V, \\
& e_{l}=r l \frac{(1-p)\left(h^{r}+l^{r}\right)^{2}+4 p h^{r} l^{r}}{4\left(h^{r}+l^{r}\right)^{2}} V .
\end{aligned}
$$

These efforts are those that, if exerted under full concealment, lead to indifference between $\mathcal{C}$ and $\mathcal{D}$. Now, using (4) and (5), we check whether these effort levels are reached for some parameter values. Hence, we rewrite (4) as

$$
\operatorname{pr} \frac{h}{4} V+(1-p) r \frac{h e_{h}^{r} e_{l}^{r}}{\left(e_{h}^{r}+e_{l}^{r}\right)^{2}} V=e_{h} .
$$

Plugging (10) into the right-hand side of (12), and after simple simplifications, we obtain the following

$$
\frac{e_{h}^{r} e_{l}^{r}}{\left(e_{h}^{r}+e_{l}^{r}\right)^{2}}=\frac{h^{r} l^{r}}{\left(h^{r}+l^{r}\right)^{2}} .
$$

\footnotetext{
17 Remark: the fact that (8) and (9) are sufficient for $\pi^{\mathcal{D}-\mathcal{C}}=0$ could have already been noticed in (7), but we needed to use (6) to show that they are also necessary for $\pi^{\mathcal{D}-\mathcal{C}}=0$.
} 
Finally, we plug (10) and (11) where we defined $J=p\left(h^{r}+l^{r}\right)^{2}+4(1-p) h^{r} l^{r}$ and $K=(1-p)\left(h^{r}+l^{r}\right)^{2}+4 p h^{r} l^{r}$ into (13), and obtain

$$
\begin{aligned}
\frac{h^{r} l^{r} J^{r} K^{r}}{\left(h^{r} J^{r}+l^{r} K^{r}\right)^{2}} & =\frac{h^{r} l^{r}}{\left(h^{r}+l^{r}\right)^{2}} \\
& \Longleftrightarrow l^{2 r} K^{r}\left(J^{r}-K^{r}\right)=h^{2 r} J^{r}\left(J^{r}-K^{r}\right),
\end{aligned}
$$

and the unique solution of (14) is $J=K$, which is equivalent to

$$
\begin{aligned}
p\left(h^{r}+l^{r}\right)^{2}+4(1-p) h^{r} l^{r} & =(1-p)\left(h^{r}+l^{r}\right)^{2}+4 p h^{r} l^{r} \\
& \Longleftrightarrow 4(1-2 p) h^{r} l^{r}=(1-2 p)\left(h^{r}+l^{r}\right)^{2}
\end{aligned}
$$

whose unique solution is $p=0.5$. Similar algebra shows that (10), (11) and $p=0.5$ satisfy (5) - besides satisfying (4) as proved. Hence, we proved that there are only three values of $p$ for which $\pi^{\mathcal{D}-\mathcal{C}}=0: 0,0.5$, and 1 .

Step 2. We write the system (4) and (5) as a unique equation in terms of $e_{h}$ and parameters only, and then we make use of the implicit function theorem to evaluate the derivative of $\pi^{\mathcal{D}-\mathcal{C}}$ in $p=0.5$, and prove that it is strictly positive. That is,

$$
\left.\frac{\partial \pi^{\mathcal{D}-\mathcal{C}}}{\partial p}\right|_{p=0.5}>0 .
$$

Remember that efforts under $\mathcal{D}$ are not functions of $p$, unlike the efforts under $\mathcal{C}$. We omitted this detail so far in the notation, and we now write it when it would otherwise yield confusion as we need to differentiate with respect to $p$.

To simplify $\pi^{\mathcal{D}-\mathcal{C}}$ we use the same steps used to move from (7) to (8), where we simplified a $p$, and get

$$
\begin{aligned}
\left.\frac{\partial}{\partial p}\left[p^{2} e_{h h}+p(1-p) e_{h l}-p e_{h}(p)\right]\right|_{p=0.5} & >0 \\
{\left.\left[2 p e_{h h}+e_{h l}-2 p e_{h l}-e_{h}(p)-p \frac{\partial e_{h}(p)}{\partial p}\right]\right|_{p=0.5} } & >0 \\
e_{h h}-e_{h}(0.5) & >\frac{1}{2}\left(\left.\frac{\partial e_{h}(p)}{\partial p}\right|_{p=0.5}\right) .
\end{aligned}
$$

When $p=0.5$, we know from Step 1 that $\pi^{\mathcal{D}-\mathcal{C}}=0$, and hence from $(8), e_{h}(0.5)=$ $\frac{e_{h h}+e_{h l}}{2}$. Therefore, (15) is equivalent to

$$
e_{h h}-e_{h l}>\left.\frac{\partial e_{h}(p)}{\partial p}\right|_{p=0.5}
$$


The left-hand side of (16) is known by (3). The right-hand side is trickier. First, isolate $e_{l}$ in (6) and use $e_{h h}$ and $e_{l l}$ from (3) to obtain:

$$
e_{l}=\frac{4 p l e_{h}+(1-2 p) r l h V}{4(1-p) h} .
$$

Use (17) into (4), and obtain

$$
f\left(e_{h}, p\right) \equiv p \frac{r}{4 e_{h}} V+4^{r} h^{r} l^{r} r \frac{(1-p)^{r+1} e_{h}^{r-1}\left[h(1-2 p) V+4 p e_{h}\right]^{r}}{\left[4^{r} h^{r}(1-p)^{r} e_{h}^{r}+\left(4 p l e_{h}+h l(1-2 p) V\right)^{r}\right]^{2}}-\frac{1}{h}=0 .
$$

The defined $f\left(e_{h}, p\right)$ is an equation in $p$ and $e_{h}$ only, and hence by the implicit function theorem

$$
\left.\frac{\partial e_{h}(p)}{\partial p}\right|_{p=0.5}=-\left.\frac{\left.\frac{\partial f\left(e_{h}, p\right)}{\partial p}\right|_{p=0.5}}{\frac{\partial f\left(e_{h}, p\right)}{\partial e_{h}}}\right|_{p=0.5}
$$

We will eventually plug (18) into (16) to conclude the proof of Step 2. We start with the denominator of (18):

$$
\begin{aligned}
\left.\frac{\partial f\left(e_{h}, p\right)}{\partial e_{h}}\right|_{p=0.5} & =\frac{\partial f\left(e_{h}, 0.5\right)}{\partial e_{h}} \\
& =\left.\frac{\partial}{\partial e_{h}}\left[\frac{r}{8 e_{h}} V+\frac{h^{r} l^{r} r}{2\left(h^{r}+l^{r}\right)^{2} e_{h}} V\right]\right|_{p=0.5} \\
& =-\left.\frac{r}{e_{h}^{2}}\left[\frac{h^{2 r}+l^{2 r}+6 h^{r} l^{r}}{8\left(h^{r}+l^{r}\right)^{2}} V\right]\right|_{p=0.5}
\end{aligned}
$$

Note that when $p=0.5$ equilibrium effort is $e_{h}=r h \frac{h^{2 r}+l^{2 r}+6 h^{r} l^{r}}{8\left(h^{r}+l^{r}\right)^{2}} V$. This is easy to verify, since $p=0.5$ corresponds to the contest of Malueg and Yates (2004)—see their expression (20). We use this expression into (19) and obtain

$$
\left.\frac{\partial f\left(e_{h}, p\right)}{\partial e_{h}}\right|_{p=0.5}=-\left.\frac{1}{h e_{h}}\right|_{p=0.5}
$$


Hence, expression (18) reads

$$
\begin{aligned}
\left.\frac{\partial e_{h}(p)}{\partial p}\right|_{p=0.5} & =-\frac{\left.\frac{\partial f\left(e_{h}, p\right)}{\partial p}\right|_{p=0.5}}{\left.\frac{\partial f\left(e_{h}, p\right)}{\partial e_{h}}\right|_{p=0.5}} \\
& =\left(\left.h e_{h} \frac{\partial f\left(e_{h}, p\right)}{\partial p}\right|_{p=0.5}\right) \\
& =\frac{r h}{4} V+4^{r} h^{r+1} l^{r} r e_{h}^{r} \frac{\partial}{\partial p} \\
& {\left.\left[\frac{(1-p)^{r+1}\left[h(1-2 p) V+4 p e_{h}\right]^{r}}{\left[4^{r} h^{r}(1-p)^{r} e_{h}^{r}+\left(4 p l e_{h}+h l(1-2 p) V\right)^{r}\right]^{2}}\right]\right|_{p=0.5} } \\
= & \frac{r h}{4} V+\left.4^{r} h^{r+1} l^{r} r e_{h}^{r} \frac{\partial}{\partial p}\left[\frac{a(p) b(p)}{[c(p)]^{2}}\right]\right|_{p=0.5}
\end{aligned}
$$

where we defined

$$
\begin{aligned}
& a(p)=(1-p)^{r+1}, \\
& b(p)=\left[h(1-2 p) V+4 p e_{h}\right]^{r}, \\
& c(p)=4^{r} h^{r}(1-p)^{r} e_{h}^{r}+\left(4 p l e_{h}+h l(1-2 p) V\right)^{r} .
\end{aligned}
$$

Hence,

$$
\left.\frac{\partial}{\partial p}\left[\frac{a(p) b(p)}{[c(p)]^{2}}\right]\right|_{p=0.5}=\left.\frac{a^{\prime}(p) b(p)+a(p) b^{\prime}(p)}{[c(p)]^{2}}\right|_{p=0.5}-\left.\frac{2 a(p) b(p) c^{\prime}(p)}{[c(p)]^{3}}\right|_{p=0.5}
$$

From the definitions of the functions $a, b$ and $c$ compute their values and their derivatives when $p=0.5$.

$$
\begin{array}{ll}
a(0.5)=\frac{1}{2^{r+1}} & a^{\prime}(0.5)=-\frac{r+1}{2^{r}} \\
b(0.5)=2^{r} e_{h}^{r} & b^{\prime}(0.5)=2^{r+1} r e_{h}^{r-1}\left[e_{h}-h V / 2\right] \\
c(0.5)=2^{r} e_{h}^{r}\left(h^{r}+l^{r}\right) & c^{\prime}(0.5)=-2^{r+1} h^{r} r e_{h}^{r}+2^{r+1} l^{r} r e_{h}^{r-1}\left(e_{h}-h V / 2\right)
\end{array}
$$


Plug these results into (21) to write (20) in the following way ${ }^{18}$

$$
\begin{aligned}
\left.\frac{\partial e_{h}(p)}{\partial p}\right|_{p=0.5}= & \frac{r h}{4} V+ \\
& -4^{r} h^{r+1} l^{r} r e_{h}^{r}\left[\frac{e_{h}^{r}(r+1)-r e_{h}^{r-1}\left(e_{h}-h V / 2\right)}{\left[2^{r} e_{h}^{r}\left(h^{r}+l^{r}\right)\right]^{2}}\right. \\
& \left.\quad+\frac{r e_{h}^{r} 2^{r+1}\left[-h^{r} e_{h}^{r}+l^{r} e_{h}^{r-1}\left(e_{h}-h V / 2\right)\right]}{\left[2^{r} e_{h}^{r}\left(h^{r}+l^{r}\right)\right]^{3}}\right] \\
= & \frac{r h}{4} V+4 h^{r+1} l^{r} r \frac{l^{r}-h^{r}}{\left(h^{r}+l^{r}\right)\left[\left(h^{r}+l^{r}\right)^{2}+4 h^{r} l^{r}\right]} V \\
& -h^{r+1} l^{r} r \frac{l^{r}(2 r+1)+h^{r}(1-2 r)}{\left(h^{r}+l^{r}\right)^{3}} V,
\end{aligned}
$$

where we used the fact that $e_{h}=r h \frac{h^{2 r}+l^{2 r}+6 h^{r} l^{r}}{8\left(h^{r}+l^{r}\right)^{2}} V$ when $p=0.5$.

Therefore, we can finally evaluate expression (16).

$$
\begin{aligned}
\frac{r h}{4}-r h \frac{h^{r} l^{r}}{\left(h^{r}+l^{r}\right)^{2}}> & \frac{r h}{4}+4 h^{r+1} l^{r} r \frac{l^{r}-h^{r}}{\left(h^{r}+l^{r}\right)\left[\left(h^{r}+l^{r}\right)^{2}+4 h^{r} l^{r}\right]}+ \\
& -h^{r+1} l^{r} r \frac{l^{r}(2 r+1)+h^{r}(1-2 r)}{\left(h^{r}+l^{r}\right)^{3}} \\
\Longleftrightarrow & 2 r \frac{l^{r}-h^{r}}{\left(h^{r}+l^{r}\right)^{2}}>4 \frac{l^{r}-h^{r}}{\left[\left(h^{r}+l^{r}\right)^{2}+4 h^{r} l^{r}\right]} \\
\Longleftrightarrow & 2\left(h^{r}+l^{r}\right)^{2}>r\left[\left(h^{r}+l^{r}\right)^{2}+4 h^{r} l^{r}\right] .
\end{aligned}
$$

By $r \leq 1$, it suffices to show that

$$
\begin{aligned}
2\left(h^{r}+l^{r}\right)^{2} & >\left(h^{r}+l^{r}\right)^{2}+4 h^{r} l^{r} \\
& \Longleftrightarrow\left(h^{r}-l^{r}\right)^{2}>0,
\end{aligned}
$$

and the result follows.

Step 3. The continuity of $e_{h}$ and $e_{l}$ in $p$ directly follows from the Maximum Theorem applied to contestants' payoff, which is continuous and strictly concave in own effort. The continuity of $\pi^{\mathcal{D}-\mathcal{C}}$ in $p$ follows from the continuity of $e_{h}$ and $e_{l}$.

\section{Proof of Theorem 2 and Proposition 3}

If $p \in\{0,1\}$, the principal is trivially indifferent between all disclosure policies. The ranking between $\pi^{\{\mathcal{D}, \mathcal{D}, \mathcal{D}\}}$ and $\pi^{\{\mathcal{C}, \mathcal{C}, \mathcal{C}\}}$ is known from Theorem 1. Hence, in what follows, we focus on $p \in(0,1)$ and on all rankings except the one between $\pi^{\{\mathcal{D}, \mathcal{D}, \mathcal{D}\}}$ and $\pi^{\{\mathcal{C}, \mathcal{C}, \mathcal{C}\}}$.

18 For the sake of brevity, we use $e_{h}$ rather than $\left.e_{h}\right|_{p=\frac{1}{2}}$. 
First, we spell out some Preliminaries, including in Lemma 5 two key monotonicities of efforts in beliefs. Second, building on Preliminaries and Lemma 5, we prove Theorem 2 in three steps. Denoting by $\pi^{\mathcal{P}}$ the expected sum of efforts under disclosure policy $\mathcal{P}$, we prove that $\pi^{\{\mathcal{D}, \mathcal{D}, \mathcal{D}\}}>\pi^{\{\mathcal{C}, \mathcal{C}, \mathcal{D}\}}\left(\right.$ Step 1), that $\pi^{\{\mathcal{D}, \mathcal{C}, \mathcal{C}\}}>\pi^{\{\mathcal{D}, \mathcal{D}, \mathcal{D}\}}$ (Step 2), and that $\pi^{\{\mathcal{D}, \mathcal{C}, \mathcal{C}\}}>\pi^{\{\mathcal{C}, \mathcal{C}, \mathcal{C}\}}($ Step 3). Finally, note that in all the remaining policies (i.e., $\{\mathcal{C}, \mathcal{D}, \mathcal{D}\},\{\mathcal{D}, \mathcal{C}, \mathcal{D}\},\{\mathcal{D}, \mathcal{D}, \mathcal{C}\},\{\mathcal{C}, \mathcal{D}, \mathcal{C}\})$ contestants perfectly infer types, and thus they are outcome-equivalent to $\mathcal{P}=\{\mathcal{D}, \mathcal{D}, \mathcal{D}\}$. Theorem 2 thus follows from the three steps.

Finally, in order to prove Proposition 3, notice the Steps 1-2 imply $\pi^{\{\mathcal{D}, \mathcal{C}, \mathcal{C}\}}>$ $\pi^{\{\mathcal{D}, \mathcal{D}, \mathcal{D}\}}>\pi^{\{\mathcal{C}, \mathcal{C}, \mathcal{D}\}}$, and Step 3 implies that $\{\mathcal{C}, \mathcal{C}, \mathcal{C}\}$ is not the best policy. In the additional Step 4 below we prove that $\{\mathcal{C}, \mathcal{C}, \mathcal{C}\}$ is not the worst policy either. By Theorem 1 the full ranking of Corollary 4 then follows.

Preliminaries First, we derive two key equilibrium properties, (22) and (23), which hold for the two new disclosure policies, $\{\mathcal{C}, \mathcal{C}, \mathcal{D}\}$ and $\{\mathcal{D}, \mathcal{C}, \mathcal{C}\}$, by plugging the appropriate $p$.

In particular, for policy $\{\mathcal{C}, \mathcal{C}, \mathcal{D}\}$ we plug $p=1$ in (5), as a low-type observing $\mathcal{C}$ under policy $\mathcal{P}=\{\mathcal{C}, \mathcal{C}, \mathcal{D}\}$ is sure she is up against a high-type, and following the same steps used to derive (6) from (4) and (5) we obtain

$$
4 l e_{h}-4(1-p) h e_{l}=p r h l V
$$

and similarly, for policy $\{\mathcal{D}, \mathcal{C}, \mathcal{C}\}$, we plug $p=0$ in (4), as a high-type observing $\mathcal{C}$ under policy $\mathcal{P}=\{\mathcal{D}, \mathcal{C}, \mathcal{C}\}$ is sure she is up against a low-type, and following the same steps used to derive (6) from (4) and (5) we obtain

$$
4 h e_{l}-4 l p e_{h}=(1-p) r h l V .
$$

The second preliminary result is the following key lemma.

Lemma 5 The equilibrium effort of the low-type decreases in the high-type's belief of being in an even contest.

The equilibrium effort of the high-type increases in the low-type's belief of being in an even contest.

Proof of Lemma 5 For the sake of the proof of this lemma only, we denote by $p_{h}\left(p_{l}\right)$ the belief of a high (low) type of being in an even contest; that is, of being against another high (low) type. Hence, we rewrite the system of FOCs (4) and (5) as

$$
\left\{\begin{array}{l}
p_{h} A+\left(1-p_{h}\right) B=\frac{1}{h} \\
p_{l} C+\left(1-p_{l}\right) D=\frac{1}{l}
\end{array}\right.
$$

where we define

$$
A \equiv \frac{r}{4 e_{h}} V, B \equiv \frac{r e_{h}^{r-1} e_{l}^{r}}{\left[e_{h}^{r}+e_{l}^{r}\right]^{2}} V, C \equiv \frac{r}{4 e_{l}} V, D \equiv \frac{r e_{l}^{r-1} e_{h}^{r}}{\left[e_{h}^{r}+e_{l}^{r}\right]^{2}} V .
$$

We prove the statements of the lemma building on five intermediary results. 
First, we show that

$$
e_{h}>e_{l}
$$

Assume by contradiction that $e_{h} \leq e_{l}$. Since $\frac{1}{h}<\frac{1}{l}$, (24) implies

$$
p_{h} A+\left(1-p_{h}\right) B<p_{l} C+\left(1-p_{l}\right) D \text {. }
$$

Also, it is routine to show that $A \geq B, A \geq C, C \geq D$ and $B \geq D$. Thus, $A \geq$ $\max \{B, C\} \geq \min \{B, C\} \geq D$. If $B \geq C$, then a contradiction is immediately reached by $A \geq B \geq C \geq D$ and (26). If instead $C>B$, then using the definitions of $B$ and $C$ and cross-multiplying, we obtain $\left[e_{h}^{r}+e_{l}^{r}\right]^{2}>4 e_{l}^{r+1} e_{h}^{r-1}$, which requires $\left[e_{h}^{r}+e_{l}^{r}\right]^{2}>4 e_{l}^{2 r}$, which in turn can be written as $\left[e_{h}^{r}+3 e_{l}^{r}\right]\left[e_{h}^{r}-e_{l}^{r}\right]>0$. This contradicts $e_{h} \leq e_{l}$.

Second, we show that

$$
\frac{\partial\left[p_{h} A+\left(1-p_{h}\right) B\right]}{\partial e_{h}} \leq 0 \leq \frac{\partial\left[p_{h} A+\left(1-p_{h}\right) B\right]}{\partial e_{l}} .
$$

Result (27) has an easy economic interpretation; a high type's marginal payoff from higher effort is decreasing in $e_{h}$ (as the payoff function is concave), and is increasing in $e_{l}$ for $e_{l}<e_{h}$ (encouragement effect). In fact, $A$ decreases in $e_{h} . B$ decreases in $e_{h}$ because its numerator decreases in $e_{h}$ and its denominator increases in $e_{h}$. $A$ is constant in $e_{l} . B$ increases in $e_{l}$ because

$$
\begin{aligned}
\frac{\partial B}{\partial e_{l}} & =r e_{h}^{r-1} \frac{r e_{l}^{r-1}\left[e_{h}^{r}+e_{l}^{r}\right]-2 r e_{l}^{2 r-1}}{\left[e_{h}^{r}+e_{l}^{r}\right]^{3}} V \\
& =r^{2} e_{h}^{r-1} e_{l}^{r-1} \frac{e_{h}^{r}-e_{l}^{r}}{\left[e_{h}^{r}+e_{l}^{r}\right]^{3}} V>0,
\end{aligned}
$$

where the last inequality follows from (25).

Third, as can be proved with similar, thus omitted, steps as for (27),

$$
\frac{\partial\left[p_{l} C+\left(1-p_{l}\right) D\right]}{\partial e_{h}} \leq 0, \frac{\partial\left[p_{l} C+\left(1-p_{l}\right) D\right]}{\partial e_{l}} \leq 0 .
$$

Result (28) has an analogous economic interpretation as the one provided for (27).

Fourth, we show that efforts are smaller than their complete-information levels in an even contest, or formally,

$$
e_{h} \leq \frac{r h V}{4} \text { and } e_{l} \leq \frac{r l V}{4} .
$$

Consider the first equation of (24). If $p_{h}=1, e_{h}=r h V / 4$. Consider now the effect of lowering $p_{h}(<1)$. Then, by $A \geq B$, the convex combination $p_{h} A+\left(1-p_{h}\right) B$ 
decreases in $p_{h}$. To keep it equal to the constant $1 / h$, then $e_{h}$ must decrease because both $A$ and $B$ are decreasing functions of $e_{h}$, as proved above. Hence, $e_{h} \leq r h V / 4$. The proof of $e_{l} \leq r l V / 4$ is analogous.

Fifth, following the same steps used to derive (6), but for the general beliefs $p_{h}$ and $p_{l}$ as defined in this proof, we generalize (6) to

$$
\frac{\frac{4 e_{l}}{r l V}-p_{l}}{1-p_{l}}=\frac{\frac{4 e_{h}}{r h V}-p_{h}}{1-p_{h}}
$$

and recall that both $e_{l}$ and $e_{h}$ depend on $\left(p_{h}, p_{l}\right)$.

The first statement of Lemma 5 can be written as $\partial e_{l} / \partial p_{h}<0$. Assume by contradiction that $\partial e_{l} / \partial p_{h} \geq 0$. The left-hand side of (30) increases in $e_{l}$, and thus by $\partial e_{l} / \partial p_{h} \geq 0$ it also increases in $p_{h}$. Since the left-hand side of (30) increases in $p_{h}$, also the right-hand side of (30) has to increase in $p_{h}$. However, $\frac{x-p_{h}}{1-p_{h}}$ decreases in $p_{h}$ whenever $x \leq 1$ (which holds by (29)), hence the only way to have the righthand side of (30) increasing in $p_{h}$ is that $\partial e_{h} / \partial p_{h} \geq 0$. By (28), $\partial e_{l} / \partial p_{h} \geq 0$ and $\partial e_{h} / \partial p_{h} \geq 0$ lead to a contradiction. Therefore, $\partial e_{l} / \partial p_{h}<0$, proving the first statement of Lemma 5.

The second statement of Lemma 5 can be written as $\partial e_{h} / \partial p_{l}>0$. First, as we just proved, $\partial e_{l} / \partial p_{h}<0$, and hence by (28) and $p_{l} C+\left(1-p_{l}\right) D=1 / l$, it must be that $\partial e_{h} / \partial p_{h}>0$. Second, by (27) and $p_{h} A+\left(1-p_{h}\right) B=1 / h$ we obtain $\partial e_{h} / \partial p_{l}>0 \Longleftrightarrow \partial e_{l} / \partial p_{l}>0$. Therefore, the last step to finish the proof of the second statement of Lemma 5 is to assume that $\partial e_{h} / \partial p_{l} \leq 0$ and $\partial e_{l} / \partial p_{l} \leq 0$ and obtain a contradiction. For brevity, we denote these two conditions $e_{l}^{\prime} \leq 0$ and $e_{h}^{\prime} \leq 0$ in what follows. We differentiate the second equation of (24) with respect to $p_{l}$ (for the differential of $\left(1-p_{h}\right) B$ we apply the formula in (21))

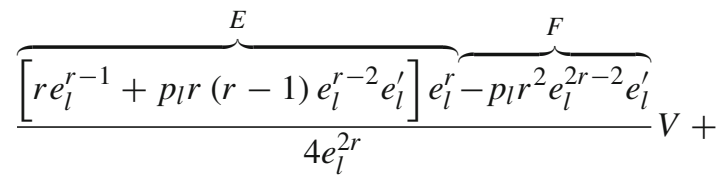

$$
\begin{aligned}
& +\frac{\overbrace{-r e_{l}^{r-1} e_{h}^{r}+\left(1-p_{l}\right)\left[r(r-1) e_{l}^{r-2} e_{h}^{r} e_{l}^{\prime}+r e_{l}^{r-1} r e_{h}^{r-1} e_{h}^{\prime}\right]}^{G}}{\left[e_{h}^{r}+e_{l}^{r}\right]^{2}} V+ \\
& \overbrace{\frac{2\left(1-p_{l}\right) r e_{l}^{r-1} e_{h}^{r}\left[r e_{h}^{r-1} e_{h}^{\prime}+r e_{l}^{r-1} e_{l}^{\prime}\right]}{\left[e_{h}^{r}+e_{l}^{r}\right]^{3}}}^{I} V=0 .
\end{aligned}
$$

We prove that $E+F+G+H+I>0$ in order to achieve a contradiction and thus end the proof. Term $F$ is trivially positive. ${ }^{19}$ Term $E+G$ is positive since it can be

19 Recall that we are under assumptions $e_{l}^{\prime} \leq 0$ and $e_{h}^{\prime} \leq 0$. 
written as

$$
E+G=\frac{r e_{l}^{2 r-1}\left[e_{h}^{r}-e_{l}^{r}\right]^{2}+p_{l} r(r-1) e_{l}^{2 r-2} e_{l}^{\prime}\left[e_{h}^{r}+e_{l}^{r}\right]^{2}}{4 e_{l}^{2 r}\left[e_{h}^{r}+e_{l}^{r}\right]^{2}}>0 .
$$

And similarly,

$$
\begin{aligned}
H+I & =\left(1-p_{l}\right) \frac{\left[r(r-1) e_{l}^{r-2} e_{h}^{r} e_{l}^{\prime}+r e_{l}^{r-1} r e_{h}^{r-1} e_{h}^{\prime}\right]\left[e_{h}^{r}+e_{l}^{r}\right]-2 r e_{l}^{r-1} e_{h}^{r}\left[r e_{h}^{r-1} e_{h}^{\prime}+r e_{l}^{r-1} e_{l}^{\prime}\right]}{\left[e_{h}^{r}+e_{l}^{r}\right]^{3}} \\
& \geq\left(1-p_{l}\right) \frac{r e_{l}^{r-1} r e_{h}^{r-1} e_{h}^{\prime}\left[e_{h}^{r}+e_{l}^{r}\right]-2 r e_{l}^{r-1} r e_{h}^{2 r-1} e_{h}^{\prime}}{\left[e_{h}^{r}+e_{l}^{r}\right]^{3}} \\
& =\left(1-p_{l}\right) r e_{l}^{r-1} r e_{h}^{r-1} e_{h}^{\prime} \frac{e_{l}^{r}-e_{h}^{r}}{\left[e_{h}^{r}+e_{l}^{r}\right]^{3}} \geq 0,
\end{aligned}
$$

where the last inequality holds true by (25) and $e_{h}^{\prime} \leq 0$. Therefore, $e_{l}^{\prime} \leq 0$ and $e_{h}^{\prime} \leq 0$ lead to a contradiction and the result follows.

Using (22), (23) and Lemma 5, we are finally ready to proceed with the four main steps needed to prove Theorem 2 and Proposition 3. The proofs of the four steps are alike. First, we simplify the difference in the expected sum of efforts under the two disclosure policies using (6), (22) and (23). Second, we use Lemma 5 to conclude the proofs.

Step 1. $\pi^{\{\mathcal{D}, \mathcal{D}, \mathcal{D}\}}>\pi^{\{\mathcal{C}, \mathcal{C}, \mathcal{D}\}}$.

In this step we denote by $e_{h}$ and $e_{l}$ the efforts under $\mathcal{C}$ and policy $\{\mathcal{C}, \mathcal{C}, \mathcal{D}\}$; that is, it is commonly known that the high-type does not know her rival's type and the low-type knows her rival's type. The claim is equivalent to

$$
\begin{array}{r}
p^{2}\left(2 e_{h h}-2 e_{h}\right)+2 p(1-p)\left(e_{h l}+e_{l h}-e_{h}-e_{l}\right)>0 \\
p e_{h h}+(1-p)\left(e_{h l}+e_{l h}\right)-(1-p) e_{l}-e_{h}>0 \\
p \frac{r h}{4} V+(1-p) r \frac{h^{r} l^{r}(h+l)}{\left(h^{r}+l^{r}\right)^{2}} V-(1-p) e_{l}-e_{h}>0
\end{array}
$$

where we used (3) in the last step. Now, use (22) to eliminate $e_{h}$, and obtain

$$
\begin{aligned}
p \frac{r h}{4} V+(1-p) r \frac{h^{r} l^{r}(h+l)}{\left(h^{r}+l^{r}\right)^{2}} V-(1-p) e_{l}-(1-p) \frac{h}{l} e_{l}-p \frac{r h}{4} V & >0 \\
r \frac{h^{r} l^{r}(h+l)}{\left(h^{r}+l^{r}\right)^{2}} V-\frac{h+l}{l} e_{l} & >0 \\
r \frac{h^{r} l^{r+1}}{\left(h^{r}+l^{r}\right)^{2}} V & >e_{l} \\
e_{l h} & >e_{l},(31)
\end{aligned}
$$

where the last step is implied by (3). 
Now, the low-type is sure she is up against a high-type in both $e_{l h}$ and $e_{l}$, but in the former the high-type's belief of being up against another high-type is 0 while in the latter it is $p$. Therefore, (31) follows from the first statement of the Lemma 5.

Step 2. $\pi^{\{\mathcal{D}, \mathcal{C}, \mathcal{C}\}}>\pi^{\{\mathcal{D}, \mathcal{D}, \mathcal{D}\}}$.

In this step we denote by $e_{h}$ and $e_{l}$ the efforts under $\mathcal{C}$ and policy $\{\mathcal{D}, \mathcal{C}, \mathcal{C}\}$; that is, it is commonly known that the high-type knows her rival's type and the low-type does not know her rival's type. The claim is equivalent to

$$
\begin{array}{r}
2 p(1-p)\left(e_{h}+e_{l}-e_{h l}-e_{l h}\right)+(1-p)^{2}\left(2 e_{l}-2 e_{l l}\right)>0 \\
e_{l}+p e_{h}-p\left(e_{h l}+e_{l h}\right)-(1-p) e_{l l}>0 \\
e_{l}+p e_{h}-p r \frac{h^{r} l^{r}(h+l)}{\left(h^{r}+l^{r}\right)^{2}} V-(1-p) \frac{r l}{4} V>0
\end{array}
$$

where we used (3) in the last step. Now, use (23) to eliminate $e_{l}$, and obtain

$$
\begin{aligned}
p \frac{l}{h} e_{h}+(1-p) \frac{r l}{4} V+p e_{h}-p r \frac{h^{r} l^{r}(h+l)}{\left(h^{r}+l^{r}\right)^{2}} V-(1-p) \frac{r l}{4} V & >0 \\
e_{h} & >r \frac{h^{r+1} l^{r}}{\left(h^{r}+l^{r}\right)^{2}} V \\
e_{h} & >e_{h l},
\end{aligned}
$$

where the last step is implied by (3).

Now, the high-type is sure she is up against a low-type in both $e_{h}$ and $e_{h l}$, but in the former the low-type's belief of being up against another low-type is $1-p$ while in the latter it is 0 . Therefore, (32) follows from the second statement of the Lemma 5.

Step 3. $\pi^{\{\mathcal{D}, \mathcal{C}, \mathcal{C}\}}>\pi^{\{\mathcal{C}, \mathcal{C}, \mathcal{C}\}}$.

Now both disclosure policies include some concealment, and hence there are two efforts under $\mathcal{C}$ for each type according to the disclosure policy. In this step, we denote by $\hat{e}_{h}$ and $\hat{e}_{l}$ the efforts under $\mathcal{C}$ and policy $\{\mathcal{D}, \mathcal{C}, \mathcal{C}\}$, that is when it is commonly known that the high-type does not know her rival's type and the low-type knows her rival's type $\left(p_{h}=0\right.$ and $\left.p_{l}=1-p\right)$, and by $\bar{e}_{h}$ and $\bar{e}_{l}$ the efforts under $\mathcal{C}$ and policy $\{\mathcal{C}, \mathcal{C}, \mathcal{C}\}$, that is when it is commonly known that no type knows her rival's type $\left(p_{h}=p\right.$ and $\left.p_{l}=1-p\right)$. The claim is equivalent to

$$
\begin{aligned}
& p^{2}\left(2 e_{h h}-2 \bar{e}_{h}\right)+ 2 p(1-p)\left(\hat{e}_{h}+\hat{e}_{l}-\bar{e}_{h}-\bar{e}_{l}\right)+(1-p)^{2}\left(2 \hat{e}_{l}-2 \bar{e}_{l}\right)>0 \\
& p^{2} \frac{r h}{4} V+p(1-p) \hat{e}_{h}-p \bar{e}_{h}+(1-p) \hat{e}_{l}-(1-p) \bar{e}_{l}>0,
\end{aligned}
$$


where we used (3). Now, use (23) for policy $\{\mathcal{D}, \mathcal{C}, \mathcal{C}\}$ to eliminate $\hat{e}_{h}$, and (6) for policy $\{\mathcal{C}, \mathcal{C}, \mathcal{C}\}$ to eliminate $\bar{e}_{h}$, and obtain

$$
\begin{aligned}
& p^{2} \frac{r h}{4} V+(1-p) \frac{h}{l} \hat{e}_{l}-(1-p)^{2} \frac{r h}{4} V-(1-p) \frac{h}{l} \bar{e}_{l}+(1-2 p) \frac{r h}{4} V \\
& \quad+(1-p) \hat{e}_{l}-(1-p) \bar{e}_{l}>0 \\
& (1-p) \frac{h}{l} \hat{e}_{l}-(1-p) \frac{h}{l} \bar{e}_{l} \\
& \quad+(1-p) \hat{e}_{l}-(1-p) \bar{e}_{l}>0 \\
& \hat{e}_{l}>\bar{e}_{l}
\end{aligned}
$$

Now, the low-type knows she is up against a high-type with probability $p$ in both $\hat{e}_{l}$ and $\bar{e}_{l}$, but in the former the high-type's belief of being up against another high-type is 0 while in the latter it is $p$. Therefore, $\hat{e}_{l}>\bar{e}_{l}$ follows from the first statement of the Lemma 5.

Step 4. $\pi^{\{\mathcal{C}, \mathcal{C}, \mathcal{C}\}}>\pi^{\{\mathcal{C}, \mathcal{C}, \mathcal{D}\}}$.

Now both disclosure policies include some concealment, and hence there are two efforts under $\mathcal{C}$ for each type according to the disclosure policy. In this step we denote by $\hat{e}_{h}$ and $\hat{e}_{l}$ the efforts under $\mathcal{C}$ and policy $\{\mathcal{C}, \mathcal{C}, \mathcal{D}\}$, that is when it is commonly known that the high-type does not know her rival's type and the low-type knows her rival's type $\left(p_{h}=p\right.$ and $\left.p_{l}=0\right)$, and by $\bar{e}_{h}$ and $\bar{e}_{l}$ the efforts under $\mathcal{C}$ and policy $\{\mathcal{C}, \mathcal{C}, \mathcal{C}\}$, that is when it is commonly known that no type knows her rival's type $\left(p_{h}=p\right.$ and $\left.p_{l}=1-p\right)$. The claim is equivalent to

$$
\begin{aligned}
& p^{2}\left(2 \hat{e}_{h}-2 \bar{e}_{h}\right)+ 2 p(1-p)\left(\hat{e}_{h}+\hat{e}_{l}-\bar{e}_{h}-\bar{e}_{l}\right)+(1-p)^{2}\left(2 e_{l l}-2 \bar{e}_{l}\right)<0 \\
&(1-p)^{2} \frac{r l}{4} V+p \hat{e}_{h}-p \bar{e}_{h}+p(1-p) \hat{e}_{l}-(1-p) \bar{e}_{l}<0
\end{aligned}
$$

where we used (3). Now, use (22) for policy $\{\mathcal{C}, \mathcal{C}, \mathcal{D}\}$ to eliminate $\hat{e}_{l}$, and (6) for policy $\{\mathcal{C}, \mathcal{C}, \mathcal{C}\}$ to eliminate $\bar{e}_{l}$, and obtain

$$
\begin{aligned}
(1-p)^{2} \frac{r l}{4} V+p \hat{e}_{h}-p \bar{e}_{h}+p \frac{l}{h} \hat{e}_{h}-p^{2} \frac{r l}{4} V-p \frac{l}{h} \bar{e}_{h}-(1-2 p) \frac{r l}{4} V & <0 \\
p \hat{e}_{h}-p \bar{e}_{h}+p \frac{l}{h} \hat{e}_{h}-p \frac{l}{h} \bar{e}_{h} & <0 \\
\bar{e}_{h} & >\hat{e}_{h}(33)
\end{aligned}
$$

Now, the high-type knows she is up against another high-type with probability $p$ in both in both $\bar{e}_{h}$ and $\hat{e}_{h}$, but in the former the low-type's belief of being up against another low-type is 0 while in the latter it is $1-p$. Therefore, (33) follows from the second statement of the Lemma 5.

Corner equilibria Finally, we are left to show that considering corner equilibria does not affect the main results. In any corner equilibria, it cannot be that $e_{h}=0$, as it would contradict $e_{h}>e_{l}$ (see (25) in the proof of Lemma 5). When $e_{l}=0$, the ranking of policies of Proposition 3, and therefore Theorem 2, are not affected. It 
could be proved that $e_{l}=0$ can be sustained in equilibrium only if $r=1$, the belief of the high-type of being up against another high-type is greater than $4 l / h$, and only when $\mathcal{P}=\{\mathcal{C}, \mathcal{C}, \mathcal{D}\}$, so that the low-type infers to be up against a high-type and the high-type believes she is up against a high-type with probability $p$. The reason why corner equilibria do not affect the results can be immediately noticed in the only two steps where disclosure policy $\{\mathcal{C}, \mathcal{C}, \mathcal{D}\}$ plays a role; the proof of Step 1 in the proof of Theorem 2, which carries over to the case of $e_{l}=0$, and the proof of Step 4 in the proof of Theorem 2, which carries over to the case of $\hat{e}_{l}=0$.

\section{Appendix B: Extensions}

In this Appendix, we test the robustness of Theorem 1 along four dimensions.

(B.1) Ex-post disclosure. We assumed the principal has ex-ante commitment power. If the principal lacks such commitment power, then she might have an incentive to renege on her ex-ante announced disclosure policy once she observes types. Hence, the disclosure policy is effectively chosen ex-post. In what follows, we claim that concealment under $\{h, h\}$ or $\{l, l\}$ is never profitable for the principal, hence concealment in the only remaining contingency $\{h, l\}$ makes contestants perfectly infer types, and thus contestants always exert their equilibrium efforts under full disclosure.

First, when the principal observes $\{h, h\}$, she is strictly better-off fully disclosing types regardless of what she announced before observing types and regardless of the contestants' beliefs if she had instead concealed types; in fact, the maximum effort for a high-type is exerted when she is certain she is up against another high-type under disclosure. Formally, $e_{h h}$ is the highest effort a high-type ever exerts (see the expression of $e_{h h}$ in (3) and (29) in the proof of Lemma 5). Thus, the principal is strict better-off disclosing upon observing $\{h, h\}$ unless $p=1$, in which case any disclosure policy reaches the maximum $e_{h h}$.

Analogously, when the principal observes $\{l, l\}$, she is also strictly better-off fully disclosing types; in fact, the maximum effort for a low-type is exerted when she is certain she is up against another low-type under disclosure (see the expression of $e_{l l}$ in (3) and (29) in the proof of Lemma 5). This is true unless $p=0$, in which case any disclosure policy reaches the maximum $e_{l l}$.

Therefore, ruling out the trivial $p \in\{0,1\}$ cases, the principal might be weakly better-off concealing types only when types are $\{h, l\}$. However, the ex-post concealment conveys itself information to contestants, because they know that the principal was aware of the realization of types when she chose to conceal. Since $\{h, l\}$ is the only contingency where the principal might conceal, contestants infer that concealment implies that types are $\{h, l\}$. Therefore, under ex-post disclosure contestants' types are always common-knowledge, and thus contestants exert $\mathcal{D}$-equilibrium efforts.

Finally, since with ex-ante commitment power the principal can always achieve the $\mathcal{D}$-equilibrium efforts, if the principal could choose to commit ex-ante to a disclosure policy, or wait to first observe types and then choose a disclosure policy, she never has an incentive to wait and observe types.

(B.2) Principal's objective function. We assumed the principal maximizes aggregate effort. There are plausible alternatives. First, if one has in mind harmful conflicts or 
wasteful lobbying, the principal minimizes aggregate effort; the ranking of policies we retrieved would then be simply inverted. Second, if one has in mind contests for hiring new employees or the economics job market, the principal aims for selection of the most skilled contestant as the winner; when the principal maximizes the probability of a high-type winner, the optimal disclosure policy is $\mathcal{D}$ if $p \in(0,0.5), \mathcal{C}$ if $p \in(0.5,1)$, and indifference if $p \in\{0,0.5,1\} .^{20}$ Third, in an architectural or research contest the principal will eventually only implement the winning project; when the principal maximizes the expected winning effort (EWE), she favours heterogeneity of efforts more than when maximizing aggregate effort (see Serena (2017) for the intuition), and situations of heterogeneity of efforts arise only in uneven contests $-\{h, l\}$ or $\{l, h\}$. Since in uneven contests the principal is better-off having committed to conceal types, EWE-maximization increases the incentive to conceal types as opposed to the standard aggregate effort maximization. In fact, the optimal disclosure policy becomes to commit to full concealment (disclosure) if $p \in[0, \bar{p}] \cup\{1\}(p \in[\bar{p}, 1])$, where $\exists ! \bar{p} \in\left(\frac{1}{2}, 1\right]$.

(B.3) Continuum of types. We assumed a binary type space and found a tight link between the relative likelihood of high- and low-types and the optimal disclosure policy (see Theorem 1). A natural question is whether this link carries over with a continuum of types. Due to the well-known lack of tractability of private information contests with a continuum of types, we rely on two specific results from the literature. First, Ewerhart (2010) finds that under a particular continuous distribution of types, the Tullock contest with $r=1$ does admit a closed-form solution for equilibrium efforts. His results can be used to show that the link between the relative likelihood of types and optimal disclosure policy that we found goes in the same direction of his special case of continuum type space. In particular, in Ewerhart's distribution lower types more likely than higher types, and in fact numerical simulations, available upon request, on Ewerhart's distribution show that the aggregate effort is greater under concealment than under disclosure, thus going in the same direction of the finding of our Theorem 1. Second, Wasser (2013), in his Table 1, provides numerical simulations of aggregate effort under complete and private information when the prior follows a Beta distribution. Wasser's numerical simulations show that if the skewness of the Beta distribution is high (low), such that the relative likelihood of higher types over that of lower types is high (low), then the aggregate effort under complete information is greater (smaller) than under private information, thus going in the same direction of the finding of our Theorem 1.

(B.4) General contest success function. We assumed $p_{i}\left(e_{i}, e_{j}\right)=e_{i}^{r} /\left(e_{i}^{r}+e_{j}^{r}\right)$ with $r \leq 1$. A generalization to $p_{i}\left(e_{i}, e_{j}\right)=f\left(e_{i}\right) /\left(f\left(e_{i}\right)+f\left(e_{j}\right)\right)$, under some carefully chosen properties of the $f$ (.) function is difficult to achieve. The reason is as follows. Indifference between disclosure and concealment is achieved when concealment- $e_{h}$ and disclosure- $e_{h}$ coincide (see Fig. 1), and the same condition holds

\footnotetext{
20 An easy way to see this is as follows. The principal's disclosure policy affects the probability of a high-type winner only when the contest is uneven, which occurs with probability $2 p(1-p)$. Thus, besides the usual indifference when $p \in\{0,1\}$, full concealment dominates full disclosure if and only if $\frac{e_{h}}{e_{h}+e_{l}} \geq \frac{e_{h l}}{e_{h l}+e_{l h}}=\frac{h}{h+l}$, or equivalently $\frac{e_{h}}{e_{l}} \geq \frac{h}{l}$. A straightforward consequence of (6) is that $\frac{e_{h}}{e_{l}} \gtreqless \frac{h}{l} \Longleftrightarrow p \gtreqless \frac{1}{2}$, and thus the result follows.
} 
for a low-type (see Fig. 2). Where and how many times do concealment- $e_{h}$ and disclosure- $e_{h}$ cross? Thanks to assumption that $f\left(e_{i}\right)=e_{i}^{r}$ with $r \leq 1$,

(A) concealment- $e_{h}$ is concave in $p$, and thus there is a unique $p \in(0,1)$ for which it equals disclosure- $e_{h}$, which is a straight line.

(B) concealment- $e_{l}$ is convex in $p$, and thus there is a unique $p \in(0,1)$ for which it equals disclosure- $e_{l}$, which is a straight line.

(C) these two unique values of $p$ both occur in $p=0.5 .^{21}$

More general standard regularity conditions on the function $f($.$) , such as$

$$
(\operatorname{Reg}): f(0)=0, f^{\prime}(.)>0 \text { and } f^{\prime \prime}(.) \leq 0
$$

guarantee none of (A)-(B)-(C), and thus it undermines the fact that $\pi^{\mathcal{D}-\mathcal{C}}=0$ in a unique point (i.e., in $p=0.5$ ). Can we find an assumption milder than $f\left(e_{i}\right)=e_{i}^{r}$ and stronger than ( $\operatorname{Reg}$ ) which would still make $\pi^{\mathcal{D}-\mathcal{C}}=0$ for a unique $p$-perhaps different than 0.5 ? Guaranteeing (A)-(B) could be easily done with a mild condition on $f^{\prime \prime \prime} .{ }^{22}$ Nevertheless, without $(\mathrm{C})$, uniqueness of $p$ for which $\pi^{\mathcal{D}-\mathcal{C}}=0$ is not granted, and $\pi^{\mathcal{D}-\mathcal{C}}$ might cross the horizontal axis several times. In fact, if say $p=0.3$ satisfies concealment $-e_{h}=$ disclosure $-e_{h}$ and $p=0.7$ satisfies concealment $-e_{l}=$ disclosure $-e_{l}$, then there could be multiple $p \in[0.3,0.7]$ such that $\pi^{\mathcal{D}-\mathcal{C}}$ changes sign.

\section{References}

Aoyagi, M.: Information feedback in a dynamic tournament. Games Econ. Behav. 70(2), 242-260 (2010) Baik, K.: Effort levels in contests with two asymmetric players. South. Econ. J. 61, 367-378 (1994)

Chen, J., Kuang, Z., Zheng, J.: Bayesian Persuasion in Sequential Tullock Contests, Working Paper (2017)

Chen, J., Kuang, Z., Zheng, J.: Optimal Joint Design of Timing and Information Disclosure in Tullock Contests, Working Paper (2018a)

Chen, J., Kuang, Z., Zheng, J.: Bayesian Persuasion in All-Pay Auction Contests, Working Paper (2018b) Denter, P., Morgan, J., Sisak, D.: Where Ignorance is Bliss, 'tis Folly to be Wise: Transparency in Contests, Working Paper (2011)

Dubey, P.: The role of information in contests. Econ. Lett. 120(2), 160-163 (2013)

Einy, E., Haimanko, O., Moreno, D., Sela, A., Shitovitz, B.: Equilibrium existence in Tullock contests with incomplete information. J. Math. Econ. 61, 241-245 (2015)

Epstein, G.S., Mealem, Y.: Who gains from information asymmetry? Theory Decis. 75(3), 305-337 (2013)

Ewerhart, C.: Rent-Seeking Contests with Independent Private Values, Working Paper (2010)

Ewerhart, C., Quartieri, F.: Unique equilibrium in contests with incomplete information. Econ. Theory 70, 1-29 (2019)

Fu, Q., Jiao, Q., Lu, J.: On disclosure policy in contests with stochastic entry. Public Choice 148(3), 419-434 (2011)

Fu, Q., Jiao, Q., Lu, J.: Disclosure policy in a multi-prize all-pay auction with stochastic abilities. Econ. Lett. 125(3), 376-380 (2014)

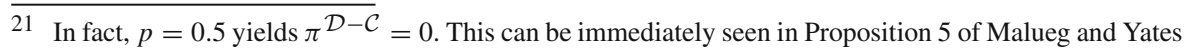
(2004).

22 In order to sign $\frac{\partial^{2} e_{h}(p)}{\partial p^{2}}$ one could differentiate (4) and (5) would with respect to $p$ twice. It is clear that it would be an expression in $f^{\prime}, f^{\prime \prime}$ and $f^{\prime \prime \prime}$. Conditions on $f^{\prime}$ and $f^{\prime \prime}$ are already imposed by (Reg). Appropriate conditions on $f^{\prime \prime \prime}$ would drive the sign of $\frac{\partial^{2} e_{h}(p)}{\partial p^{2}}$. 
Goltsman, M., Mukherjee, A.: Interim performance feedback in multistage tournaments: the optimality of partial disclosure. J. Labor Econ. 29(2), 229-265 (2011)

Heijnen, P., Schoonbeek, L.: Signaling in a rent-seeking contest with one-sided asymmetric information. J. Public Econ. Theory 66, 1-17 (2016)

Hurley, T., Shogren, J.: Asymmetric information contests. Eur. J. Polit. Econ. 14(4), 645-665 (1998a)

Hurely, T., Shogren, J.: Effort levels in a Cournot Nash contest with asymmetric information. J. Public Econ. 69, 195-210 (1998b)

Kamenica, E., Gentzkow, M.: Bayesian persuasion. Am. Econ. Rev. 101(6), 2590-2615 (2011)

Kovenock, D., Morath, F., Münster, J.: Information sharing in contests. J. Econ. Manag. Strategy 24, 570596 (2015)

Lim, W., Matros, A.: Contests with a stochastic number of players. Games Econ. Behav. 67(2), 584-597 (2009)

Loew, L.: Performance Management 2015: Coaching for Development Needed, The Brandon Hall Group (2015)

Lu, J., Ma, H., Wang, Z.: Ranking disclosure policies in all-pay auctions with incomplete information. Econ. Inquiry 56(3), 1464-1485 (2018)

Malueg, D., Yates, A.: Rent seeking with private values. Public Choice 119, 161-178 (2004)

Morgan, J., Várdy, F.: The value of commitment in contests and tournaments when observation is costly. Games Econ. Behav. 60, 326-338 (2007)

Myerson, R., Wärneryd, K.: Population uncertainty in contests. Econ. Theory 27(2), 469-474 (2006)

Nti, K.O.: Rent-seeking with asymmetric valuations. Public Choice 98(3-4), 415-430 (1999)

Serena, M.: Information in Contests, Working Paper (2014)

Serena, M.: Harnessing Beliefs to Stimulate Efforts. SSRNWorking Paper No. 2686543 (2016)

Serena, M.: Quality contests. Eur. J. Polit. Econ. 46, 15-25 (2017)

Siegel, R.: Asymmetric all-pay auctions with interdependent valuations. J. Econ. Theory 153, 684-702 (2014)

Skaperdas, S.: Contest success functions. Econ. Theory 7(2), 283-290 (1996)

The Wall Street Journal: 'Rank and Yank' Retains Vocal Fans (2012). http://www.wsj.com/news/articles/ SB10001424052970203363504577186970064375222

Tullock, G.: Efficient rent seeking. In: Buchanan, J., Tollison, R., Tullock, G. (eds.) Towards a Theory of a Rent-Seeking Society. Texas A\&M University Press, College Station (1980)

Vázquez Sedano, A.: Optimal Contest Design with Budget Constraints. Available at SSRN 2657426 (2015)

Wasser, C.: Incomplete information in rent-seeking contests. Econ. Theory 53(1), 239-268 (2013)

Wu, Z., Zheng, J.: Information sharing in private value lottery contest. Econ. Lett. 157, 36-40 (2017)

Yildirim, H.: Contests with multiple rounds. Games Econ. Behav. 51(1), 213-227 (2005)

Zhang, J., Zhou, J.: Information disclosure in contests: a Bayesian persuasion approach. Econ. J. 126(597), 2197-2217 (2016)

Publisher's Note Springer Nature remains neutral with regard to jurisdictional claims in published maps and institutional affiliations. 